Network Working Group

Request for Comments: 4475

Category: Informational
R. Sparks, Ed. Estacado Systems A. Hawrylyshen Ditech Networks A. Johnston Avaya

J. Rosenberg

Cisco Systems

H. Schulzrinne Columbia University May 2006

\title{
Session Initiation Protocol (SIP) Torture Test Messages
}

\section{Status of This Memo}

This memo provides information for the Internet community. It does not specify an Internet standard of any kind. Distribution of this memo is unlimited.

Copyright Notice

Copyright (C) The Internet Society (2006).

\section{Abstract}

This informational document gives examples of Session Initiation Protocol (SIP) test messages designed to exercise and "torture" a SIP implementation.

\section{Table of Contents}

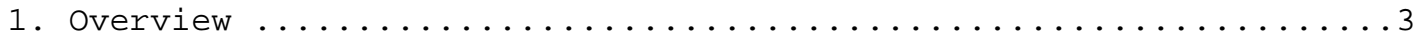

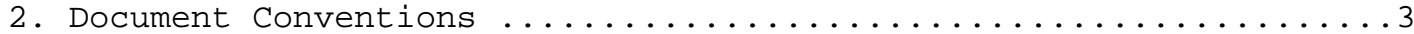

2.1. Representing Long Lines ......................

2.2. Representing Non-printable Characters .............

2.3. Representing Long Repeating Strings ................

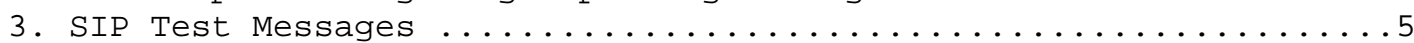

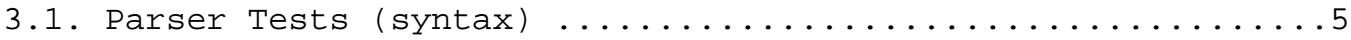

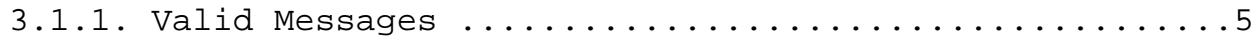

3.1.1.1. A Short Tortuous INVITE .............

3.1.1.2. Wide Range of Valid Characters ...........8

3.1.1.3. Valid Use of the $\frac{0}{0}$ Escaping Mechanism ......9

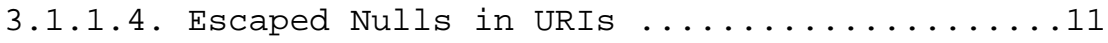

3.1.1.5. Use of $\frac{0}{0}$ When It Is Not an Escape .......11

3.1.1.6. Message with No LWS between

Display Name and $<\ldots \ldots \ldots \ldots 12$ 
3.1.1.7. Long Values in Header Fields ..........12

3.1.1.8. Extra Trailing Octets in a UDP Datagram ...14

3.1.1.9. Semicolon-Separated Parameters in

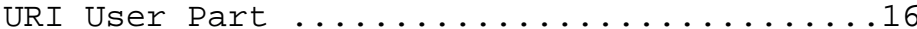

3.1.1.10. Varied and Unknown Transport Types ......16

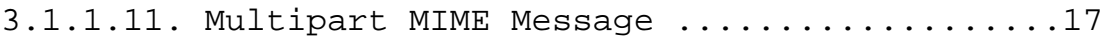

3.1.1.12. Unusual Reason Phrase ..............18

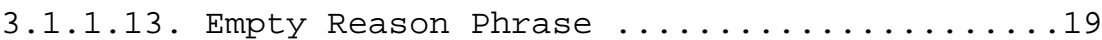

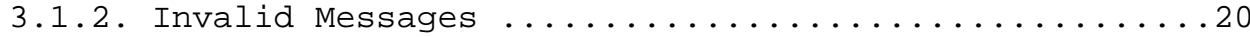

3.1.2.1. Extraneous Header Field Separators ......20

3.1.2.2. Content Length Larger Than Message .......20

3.1.2.3. Negative Content-Length ..............21

3.1.2.4. Request Scalar Fields with

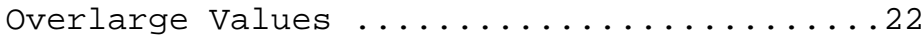

3.1.2.5. Response Scalar Fields with

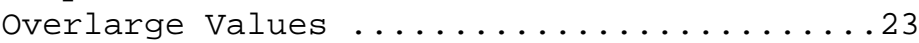

3.1.2.6. Unterminated Quoted String in

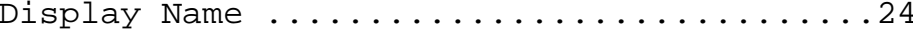

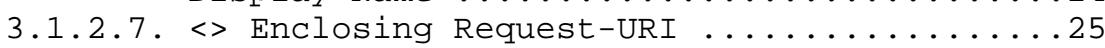

3.1.2.8. Malformed SIP Request-URI (embedded LWS) ..26

3.1.2.9. Multiple SP Separating

Request-Line Elements .................27

3.1.2.10. SP Characters at End of Request-Line ....28

3.1.2.11. Escaped Headers in SIP Request-URI ......29

3.1.2.12. Invalid Timezone in Date Header Field ...30

3.1.2.13. Failure to Enclose name-addr URI in $<>\ldots 31$

3.1.2.14. Spaces within addr-spec .................

3.1.2.15. Non-token Characters in Display Name ....32

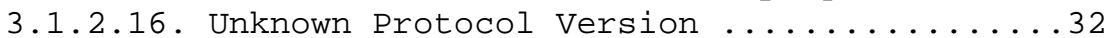

3.1.2.17. Start Line and CSeq Method Mismatch .....33

3.1.2.18. Unknown Method with CSeq Method Mismatch .33

3.1.2.19. Overlarge Response Code .............34

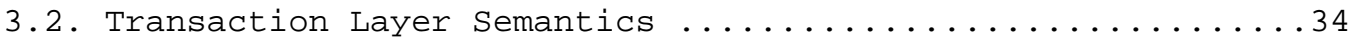

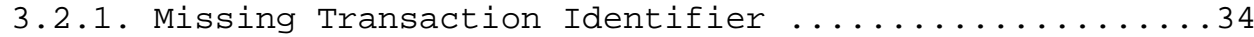

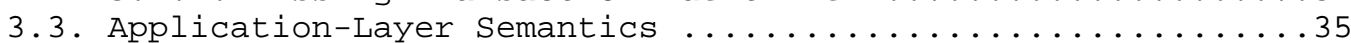

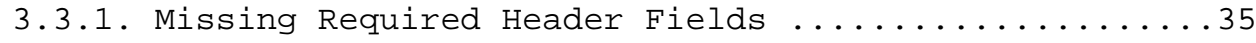

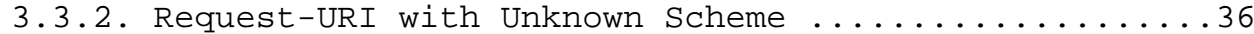

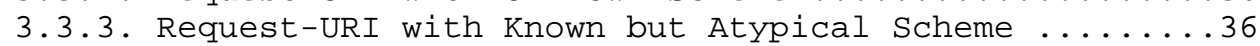

3.3.4. Unknown URI Schemes in Header Fields ..............

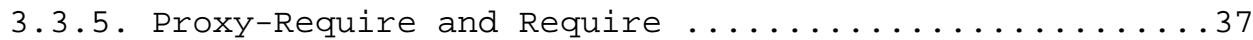

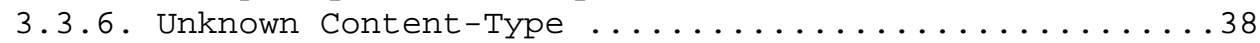

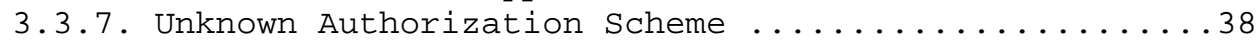

3.3.8. Multiple Values in Single Value Required Fields ....39

3.3.9. Multiple Content-Length Values ...............40

3.3.10. 200 OK Response with Broadcast Via Header

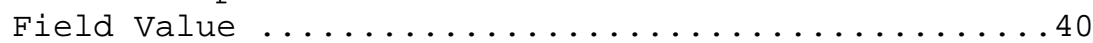

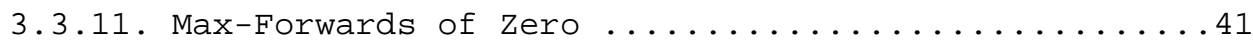

3.3.12. REGISTER with a Contact Header Parameter .......42 
3.3.13. REGISTER with a url-parameter $\ldots \ldots \ldots \ldots \ldots \ldots \ldots \ldots$

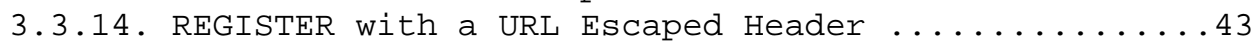

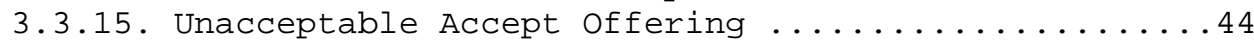

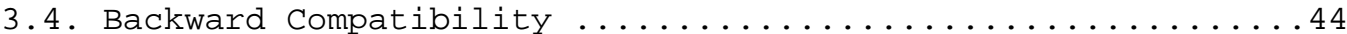

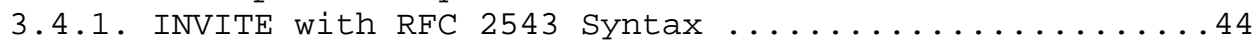

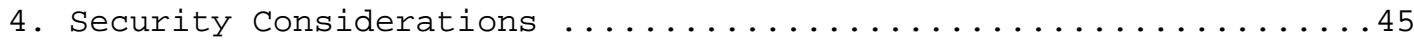

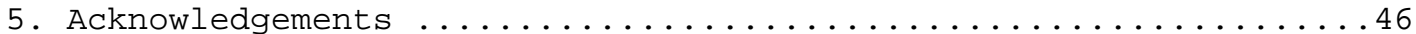

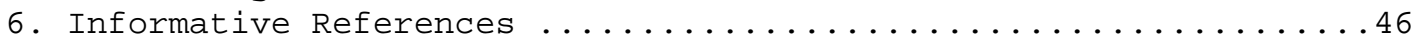

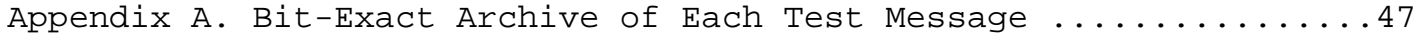

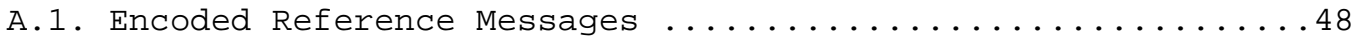

1. Overview

This document is informational and is NOT NORMATIVE on any aspect of SIP.

This document contains test messages based on the current version (2.0) of the Session Initiation Protocol as, defined in [RFC3261]. Some messages exercise SIP's use of the Session Description Protocol $(\mathrm{SDP})$, as described in [RFC3264].

These messages were developed and refined at the SIPIt interoperability test events.

The test messages are organized into several sections. Some stress only a SIP parser, and others stress both the parser and the application above it. Some messages are valid, and some are not. Each example clearly calls out what makes any invalid messages incorrect.

This document does not attempt to catalog every way to make an invalid message, nor does it attempt to be comprehensive in exploring unusual, but valid, messages. Instead, it tries to focus on areas that have caused interoperability problems or that have particularly unfavorable characteristics if they are handled improperly. This document is a seed for a test plan, not a test plan in itself.

The messages are presented in the text using a set of markup conventions to avoid ambiguity and meet Internet-Draft layout requirements. To resolve any remaining ambiguity, a bit-accurate version of each message is encapsulated in an appendix.

2. Document Conventions

This document contains many example SIP messages. Although SIP is a text-based protocol, many of these examples cannot be unambiguously rendered without additional markup due to the constraints placed on the formatting of RFCs. This document defines and uses the markup 
defined in this section to remove that ambiguity. This markup uses the start and end tag conventions of XML but does not define any XML document type.

The appendix contains an encoded binary form of all the messages and the algorithm needed to decode them into files.

\subsection{Representing Long Lines}

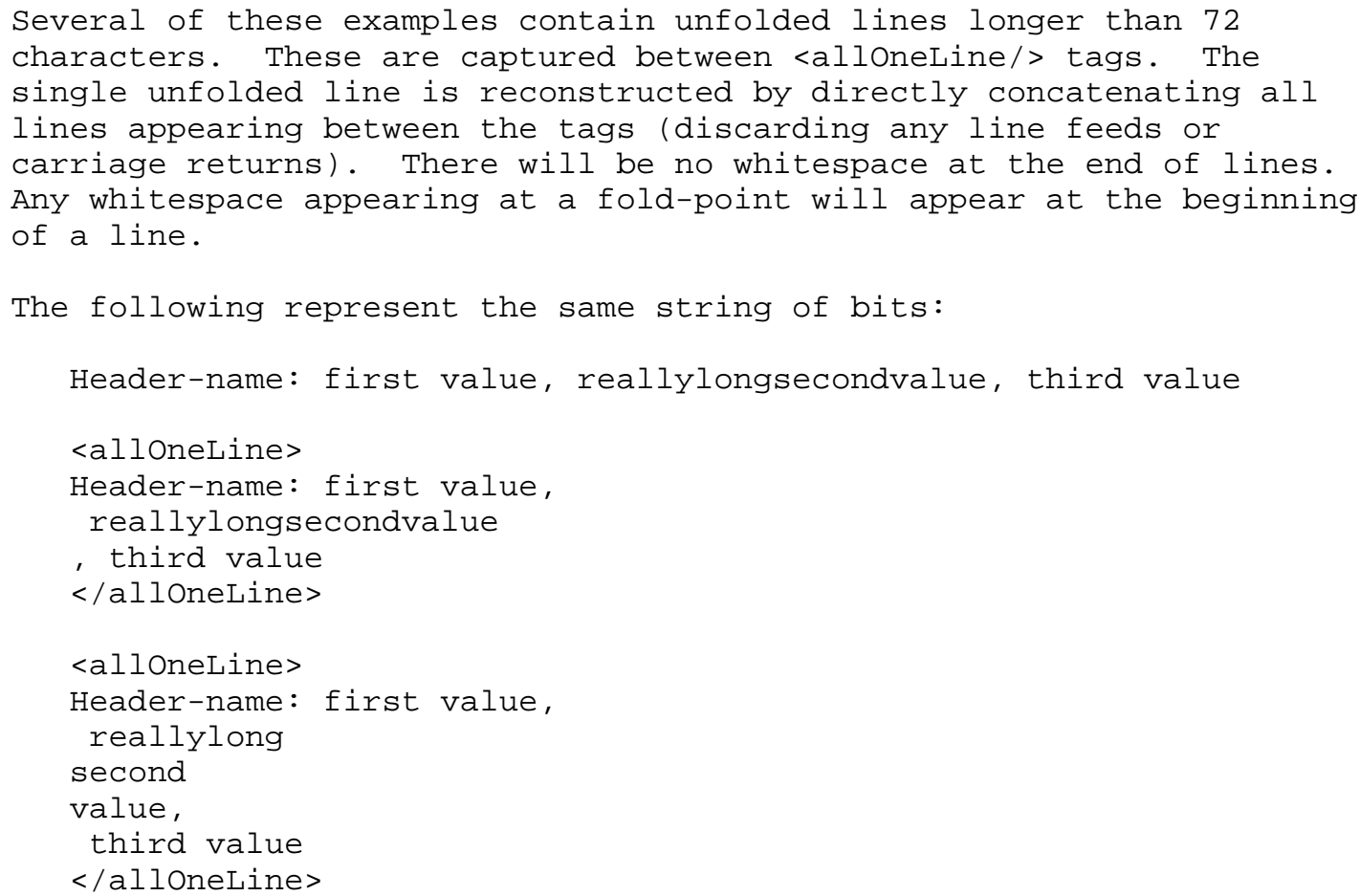

Note that this is NOT SIP header-line folding, where different strings of bits have equivalent meaning.

\subsection{Representing Non-printable Characters}

Several examples contain binary message bodies or header field values containing non-ascii range UTF-8 encoded characters. These are rendered here as a pair of hexadecimal digits per octet between <hex/> tags. This rendering applies even inside quoted-strings. 
The following represent the same string of bits:

Header-name: value one

Header-name: value $<$ hex $>206 \mathrm{~F} 6 \mathrm{E}</$ hex $>e$

The following is a subject header field containing the euro symbol:

Subject: $<$ hex $>$ E2 $82 \mathrm{AC}</$ hex $>$

2.3. Representing Long Repeating Strings

Several examples contain very large data values created with repeating bit strings. Those will be rendered here using <repeat count=some_integer $>$ value $</$ repeat $>$. As with $<$ hex $>$, this rendering applies even inside quoted strings.

For example, the value "abcabcabc" can be rendered as <repeat count $=3>$ abc $</$ repeat $>$. A display name of "1000000 bottles of beer" could be rendered as

To: " $1<$ repeat count $=6\rangle\langle$ hex $>30</$ hex $\rangle\langle/$ repeat $>$ bottles of beer" $<$ sip:beer.example.com $>$

A Max-Forwards header field with a value of one google will be rendered here as

Max-Forwards: $1<$ repeat count $=100>0</$ repeat $>$

3. SIP Test Messages

3.1. Parser Tests (syntax)

3.1.1. Valid Messages

3.1.1.1. A Short Tortuous INVITE

This short, relatively human-readable message contains:

- line folding all over.

- escaped characters within quotes.

o an empty subject.

- LWS between colons, semicolons, header field values, and other fields.

- both comma separated and separately listed header field values. 
o a mix of short and long form for the same header field name.

○ unknown Request-URI parameter.

o unknown header fields.

- an unknown header field with a value that would be syntactically invalid if it were defined in terms of generic-param.

o unusual header field ordering.

- unusual header field name character case.

- unknown parameters of a known header field.

- a uri parameter with no value.

- a header parameter with no value.

o integer fields (Max-Forwards and CSeq) with leading zeros.

All elements should treat this as a well-formed request.

The UnknownHeaderWithUnusualValue header field deserves special attention. If this header field were defined in terms of commaseparated values with semicolon-separated parameters (as would many of the existing defined header fields), this would be invalid. However, since the receiving element does not know the definition of the syntax for this field, it must parse it as a header value. Proxies would forward this header field unchanged. Endpoints would ignore the header field. 


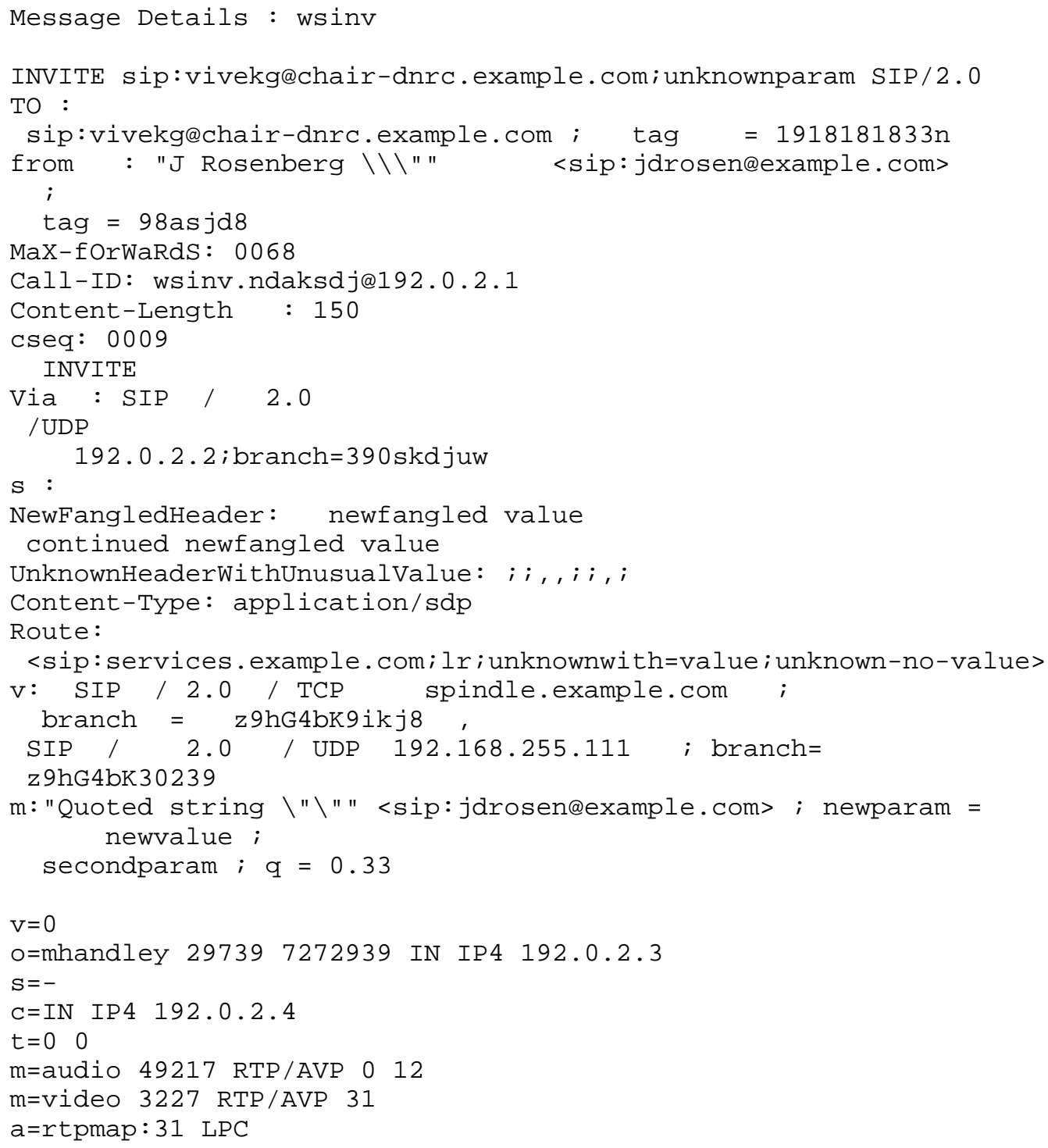




\subsubsection{Wide Range of Valid Characters}

This message exercises a wider range of characters in several key syntactic elements than implementations usually see. In particular, note the following:

- The Method contains non-alpha characters from token. Note that $\frac{0}{0}$ is not an escape character for this field. A method of INo56ITE is an unknown method. It is not the same as a method of INVITE.

- The Request-URI contains unusual, but legal, characters.

- A branch parameter contains all non-alphanum characters from token.

- The To header field value's quoted string contains quoted-pair expansions, including a quoted NULL character.

- The name part of name-addr in the From header field value contains multiple tokens (instead of a quoted string) with all non-alphanum characters from the token production rule. That value also has an unknown header parameter whose name contains the non-alphanum token characters and whose value is a non-ascii range UTF-8 encoded string. The tag parameter on this value contains the non-alphanum token characters.

- The Call-ID header field value contains the non-alphanum characters from word. Notice that in this production:

* $\%$ is not an escape character. It is only an escape character in productions matching the rule "escaped".

* " does not start a quoted string. None of ', ' or " imply that there will be a matching symbol later in the string.

* The characters []\{\}()<> do not have any grouping semantics. They are not required to appear in balanced pairs.

- There is an unknown header field (matching extension-header) with non-alphanum token characters in its name and a UTF8-NONASCII value.

If this unusual URI has been defined at a proxy, the proxy will forward this request normally. Otherwise, a proxy will generate a 404. Endpoints will generate a 501 listing the methods they understand in an Allow header field. 


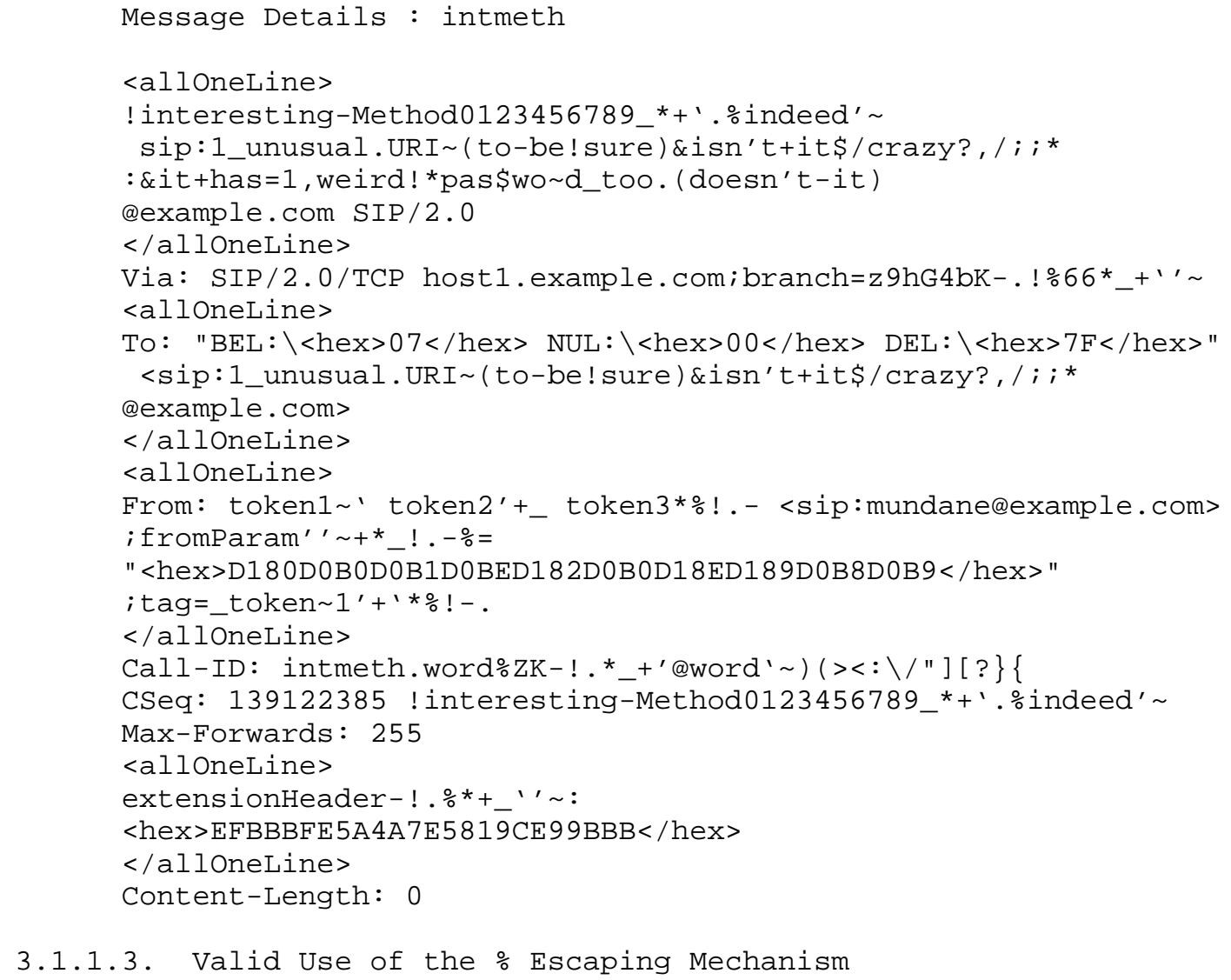

3.1.1.3. Valid Use of the ․opcaping Mechanism

This INVITE exercises the $\frac{0}{H}$ HEX HEX escaping mechanism in several places. The request is syntactically valid. Interesting features include the following:

- The request-URI has sips:userdexample.com embedded in its userpart. What that might mean to example.net is beyond the scope of this document.

- The From and To URIs have escaped characters in their userparts.

- The Contact URI has escaped characters in the URI parameters. Note that the "name" uri-parameter has a value of "value\%41", which is NOT equivalent to "valueA". Per [RFC3986], unescaping URI components is never performed recursively. 
A parser must accept this as a well-formed message. The application using the message must treat the $\frac{0}{H E X}$ HEX expansions as equivalent to the character being encoded. The application must not try to interpret $\div$ as an escape character in those places where $\% \mathrm{HEX} H E X$ ("escaped" in the grammar) is not a valid part of the construction. In [RFC3261], "escaped" only occurs in the expansions of SIP-URI, SIPS-URI, and Reason-Phrase.

Message Details : esc01

INVITE sip:sips\%3Auser\%40example.com@example.net SIP/2.0

To: sip: $\frac{\circ}{0} 5$ se 72 @example.com

From: <sip:I20have20spaces@example.net>; tag=938

Max-Forwards: 87

i: esc01.239409asdfakjkn23onasd0-3234

CSeq: 234234 INVITE

Via: SIP/2.0/UDP host5. example. net; branch=z9hG4bKkdjuw

C: application/sdp

Contact:

<sip:cal\%6Cer@host 5 . example.net; $\div 6 \mathrm{C} \div 72 ; \mathrm{n} \div 61 \mathrm{me}=\mathrm{v} \div 61 \mathrm{lue} \div 25 \div 34 \div 31>$

Content-Length: 150

$\mathrm{V}=0$

o=mhandley 297397272939 IN IP4 192.0.2.1

$\mathrm{s}=-$

$\mathrm{C}=\mathrm{IN}$ IP 4 192.0.2.1

$t=0 \quad 0$

m=audio 49217 RTP/AVP 012

$\mathrm{m}=\mathrm{video} 3227 \mathrm{RTP} / \mathrm{AVP} 31$

$a=r t$ pmap $: 31$ LPC 


\subsubsection{Escaped Nulls in URIs}

This register request contains several URIs with nulls in the userpart. The message is well formed - parsers must accept this message. Implementations must take special care when unescaping the Address-of-Record (AOR) in this request so as to not prematurely shorten the username. This request registers two distinct contact URIs.

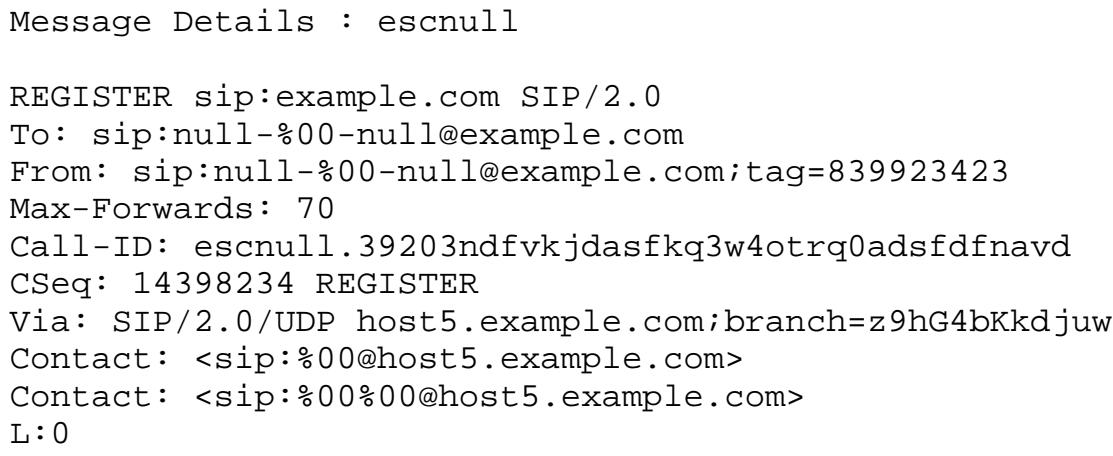

3.1.1.5. Use of $\%$ When It Is Not an Escape

In most of the places $\%$ can appear in a SIP message, it is not an escape character. This can surprise the unwary implementor. The following well-formed request has these properties:

o The request method is unknown. It is NOT equivalent to REGISTER.

- The display name portion of the To and From header fields is "ㄱㄴㄷ". Note that this is not the same as ZE.

- This message has two Contact header field values, not three. <sip:alias2@host2. example.com> is a C\%6Fntact header field value.

A parser should accept this message as well formed. A proxy would forward or reject the message depending on what the Request-URI meant to it. An endpoint would reject this message with a 501. 


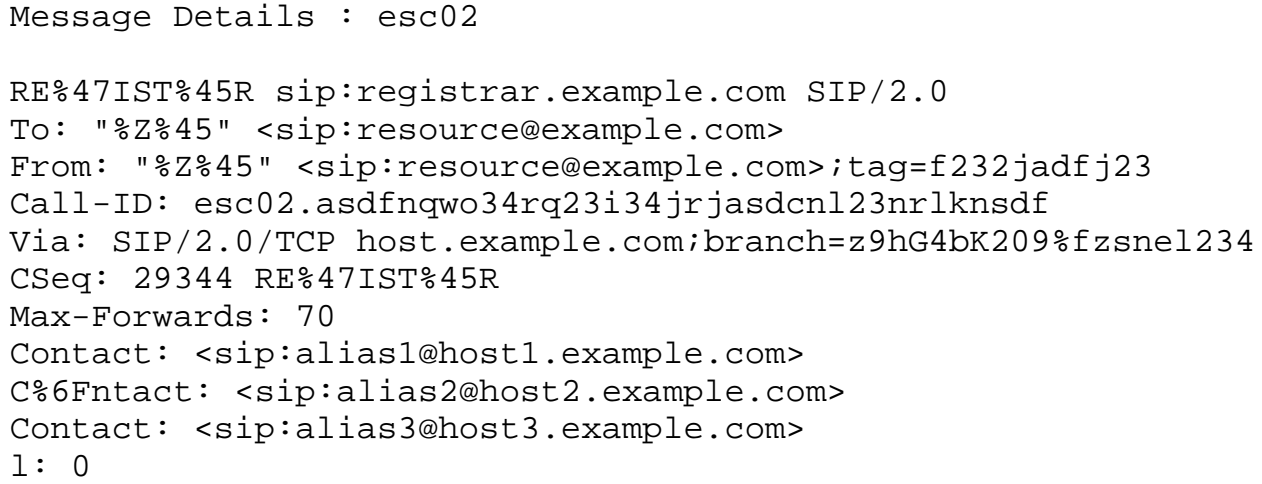

3.1.1.6. Message with No LWS between Display Name and <

This OPTIONS request is not valid per the grammar in RFC 3261 since there is no LWS between the token in the display name and < in the From header field value. This has been identified as a specification bug that will be removed when RFC 3261 is revised. Elements should accept this request as well formed.

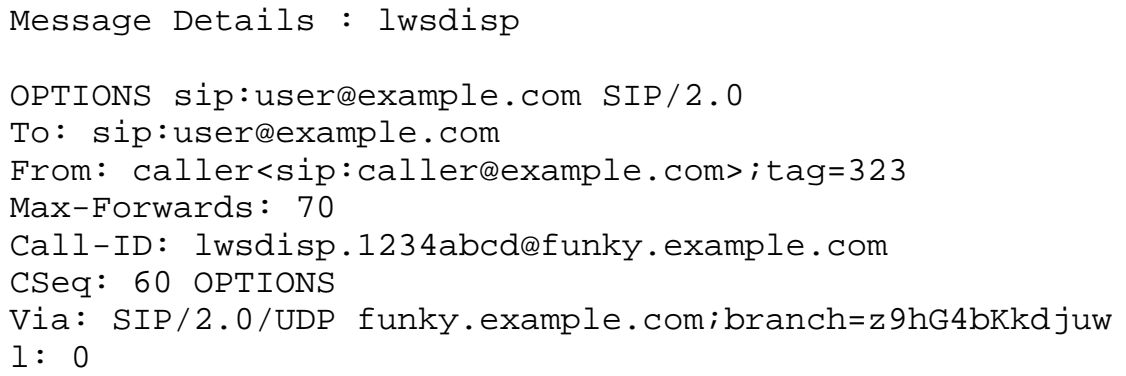

3.1.1.7. Long Values in Header Fields

This well-formed request contains header fields with many values and values that are very long. Features include the following:

- The To header field has a long display name, and long uri parameter names and values.

- The From header field has long header parameter names and values, in particular, a very long tag.

- The Call-ID is one long token. 


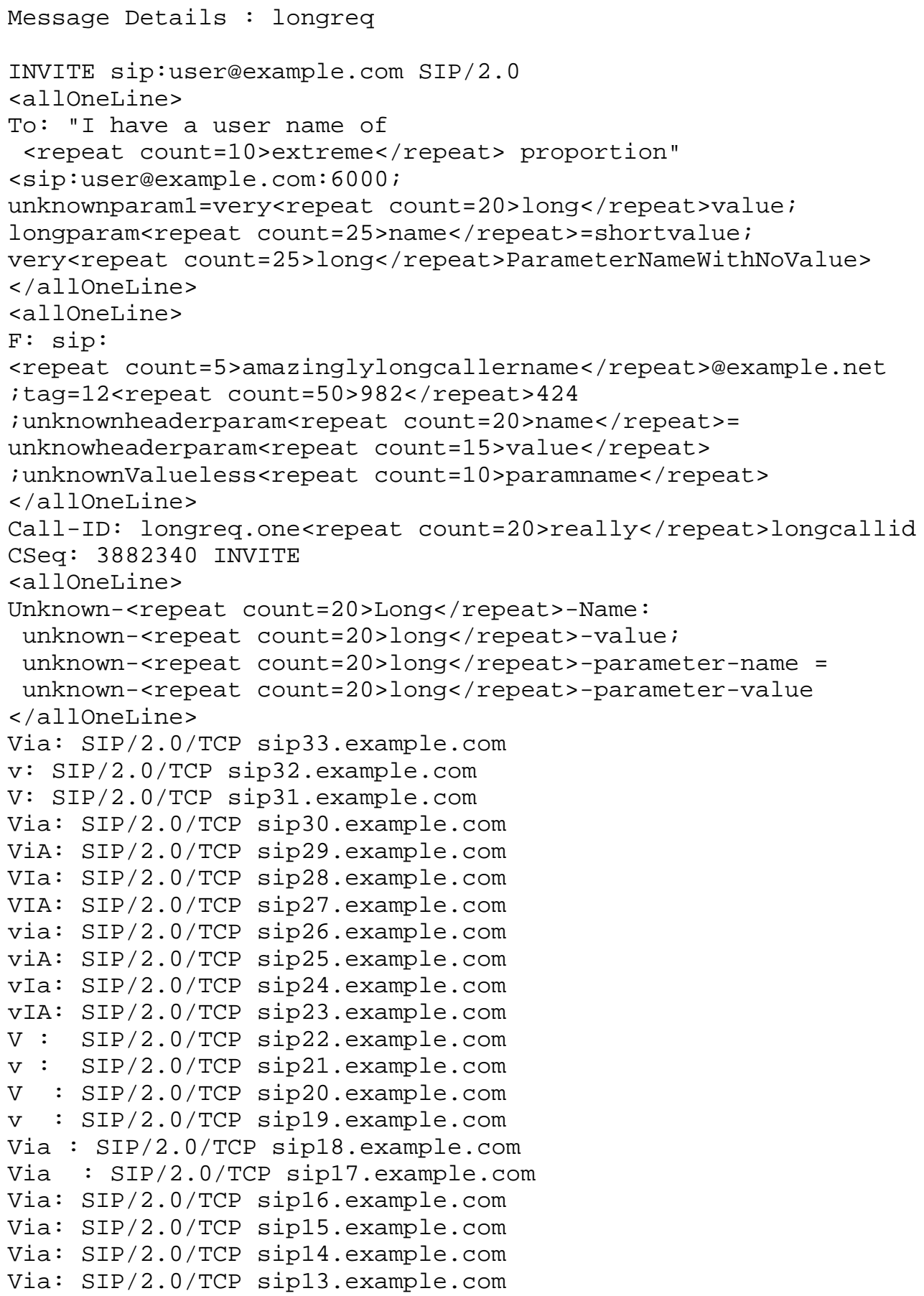




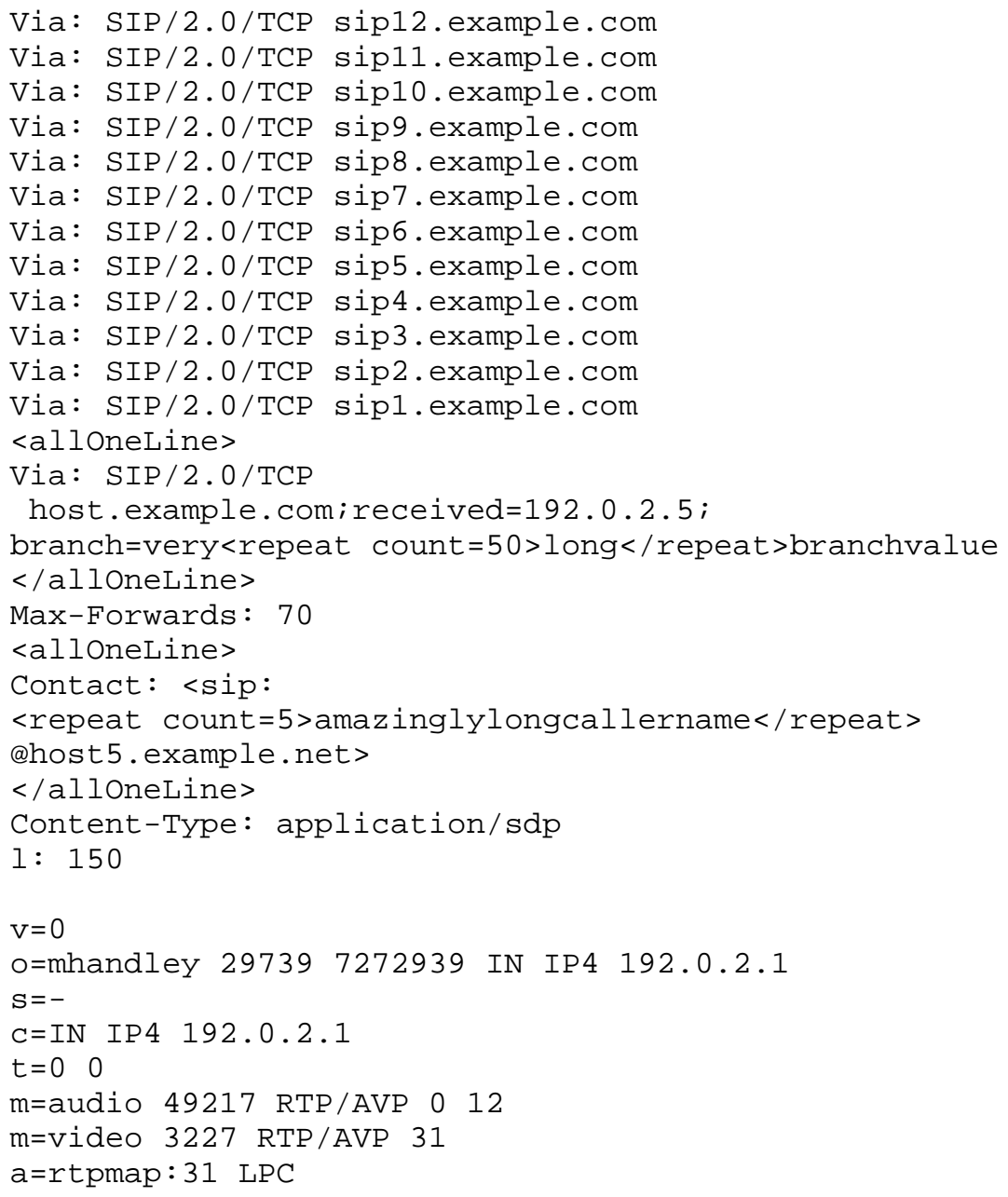

3.1.1.8. Extra Trailing Octets in a UDP Datagram

This message contains a single SIP REGISTER request, which ostensibly arrived over UDP in a single datagram. The packet contains extra octets after the body (which in this case has zero length). The extra octets happen to look like a SIP INVITE request, but (per section 18.3 of [RFC3261]) they are just spurious noise that must be ignored.

A SIP element receiving this datagram would handle the REGISTER request normally and ignore the extra bits that look like an INVITE request. If the element is a proxy choosing to forward the REGISTER, the INVITE octets would not appear in the forwarded request. 


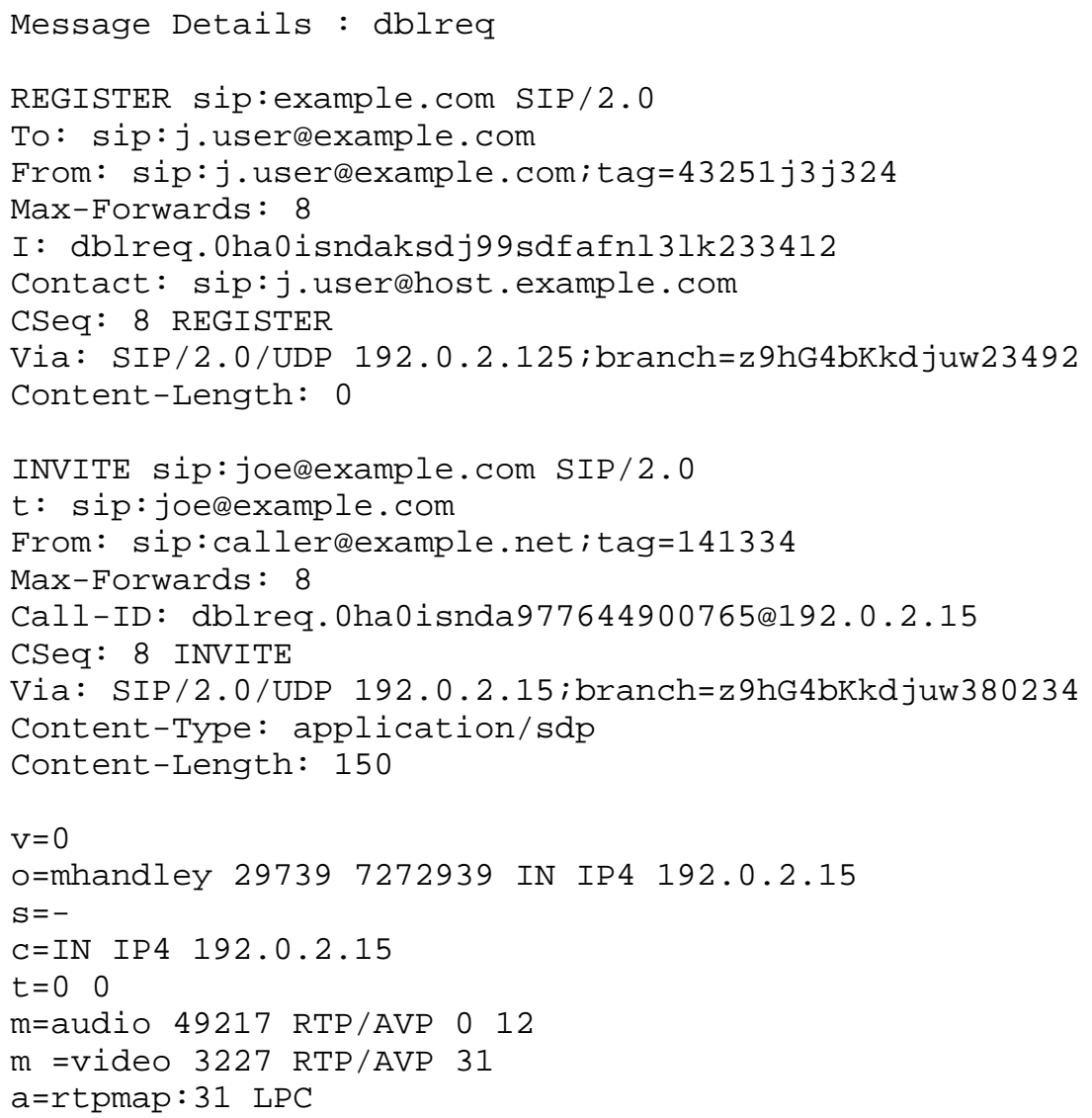




\subsubsection{Semicolon-Separated Parameters in URI User Part}

This request has a semicolon-separated parameter contained in the "user" part of the Request-URI (whose value contains an escaped a symbol). Receiving elements will accept this as a well-formed message. The Request-URI will parse so that the user part is "user; par=u@example. net".

Message Details : semiuri

OPTIONS sip:user; par=u40example.neteexample.com SIP/2.0

To: sip:j_user@example.com

From: sip:callereexample.org; tag $=33242$

Max-Forwards: 3

Call-ID: semiuri.OhaOisndaksdj

CSeq: 8 OPTIONS

Accept: application/sdp, application/pkcs7-mime, multipart/mixed, multipart/signed, message/sip, message/sipfrag

Via: SIP/2.0/UDP 192.0.2.1;branch=z9hG4bKkdjuw

$1: 0$

\subsubsection{Varied and Unknown Transport Types}

This request contains Via header field values with all known transport types and exercises the transport extension mechanism. Parsers must accept this message as well formed. Elements receiving this message would process it exactly as if the 2 nd and subsequent header field values specified UDP (or other transport).

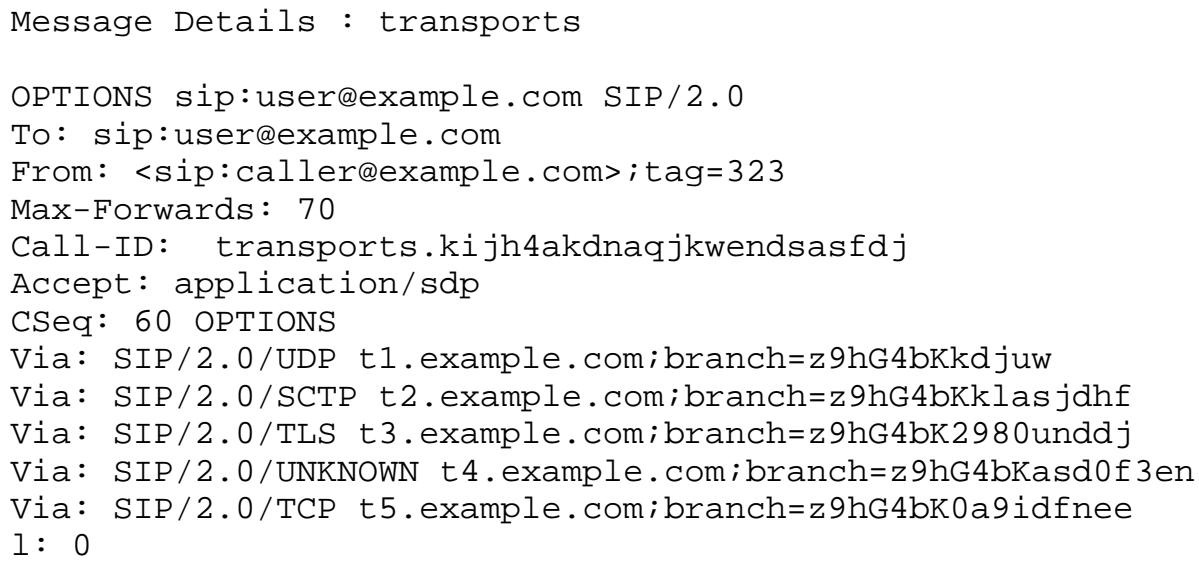




\subsubsection{Multipart MIME Message}

This MESSAGE request contains two body parts. The second part is binary encoded and contains null (0x00) characters. Receivers must take care to frame the received message properly.

Parsers must accept this message as well formed, even if the application above the parser does not support multipart/signed.

Additional examples of multipart/mime messages, in particular S/MIME messages, are available in the security call flow examples document $[\mathrm{SIP}-\mathrm{SEC}]$.

Message Details : mpart01

MESSAGE sip:kumiko@example.org SIP/2.0

<alloneLine>

Via: SIP/2.0/UDP 127.0.0.1:5070

; branch $=$ z 9hG4bK-d87543-4dade06d0bdb11ee-1--d87543-; rport

$<$ alloneLine>

Max-Forwards: 70

Route: <sip:127.0.0.1:5080>

$<$ alloneline>

Identity: r5mwreLuyDRYBi/OTiPwEsY3rEVsk/G2WxhgTV1PF7hHuL IK0YWVKZhKv9Mj8UeXqkMVbnVq37CD+813gvYjcBUaZngQmXc9WNZSDN GCzA+fWl 9MEUHWIZo1CeJebdY/XlgKeTa00lvq0rt 70Q5jis fbqMJmQF teeivUhkMWYUA=

$</$ alloneline $>$

Contact: <sip:fluffy@127.0.0.1:5070>

To: <sip:kumiko@example.org>

From: <sip:fluffy@example.com>; tag=2 fb0dcc9

Call-ID: 3d9485ad0c49859b@Zmx1ZmZ5LW1hYy0xNi5sb2NhbA. .

CSeq: 1 MESSAGE

Content-Transfer-Encoding: binary

Content-Type: multipart/mixed; boundary=7a $9 \mathrm{cbec} 02 \mathrm{ceef} 655$

Date: Sat, 15 oct 2005 04:44:56 GMT

User-Agent: SIPimp.org/0.2.5 (curses)

Content-Length: 553

--7 a 9 cbec 02 ceef 655

Content-Type: text/plain

Content-Transfer-Encoding: binary

Hello

--7 a 9 cbec 02 ceef 655

Content-Type: application/octet-stream

Content-Transfer-Encoding: binary 


\begin{abstract}
$<$ hex $>$
3082015206092 A 86

4886F70D010702A08201433082013F02

$01013109300706052 B 0 E 03021 A 300 B 06$

092A864886F70D010701318201203082

$011 \mathrm{C} 020101307 \mathrm{C} 3070310 \mathrm{~B} 3009060355$

04061302555331133011060355040813

0A43616C69666F726E69613111300F06

$03550407130853616 \mathrm{E} 204 \mathrm{~A} 6 \mathrm{~F} 7365310 \mathrm{E}$

$300 \mathrm{C} 060355040 \mathrm{~A} 130573697069743129$

3027060355040 B132053697069742054

65737420436572746966696361746520

417574686 F 7269747902080195007102

$330113300706052 \mathrm{~B} 0 \mathrm{E} 03021 \mathrm{~A} 300 \mathrm{D} 0609$

2A864886F70D01010105000481808EF 4

66F 948 F 0 522DD2E5978E9D 95AAE 9F 2FE

15A06659716292E8DA2AA8D8350A68CE

FFAE3CBD2BFF1675DDD 5648 E5 93DD 647

28F26220F7E941749E330D9A15EDABDB

93D10C42102E7B 7289D29CC0C9AE2EFB

C7C0CFF 9172F3B027E4FC027E1546DE4

B6AA3ABB3E66CCCB5DD6C64B8383149C

B8E6FF182D 944FE57B65BC99D005

$</$ hex $>$

--7 a 9 cbec 02 ceef655--
\end{abstract}

3.1.1.12. Unusual Reason Phrase

This 200 response contains a reason phrase other than "OK". The reason phrase is intended for human consumption and may contain any string produced by

Reason-Phrase $=$ * (reserved / unreserved / escaped / UTF8-NONASCII / UTF8-CONT / SP / HTAB)

This particular response contains unreserved and non-ascii UTF-8 characters. This response is well formed. A parser must accept this message. 


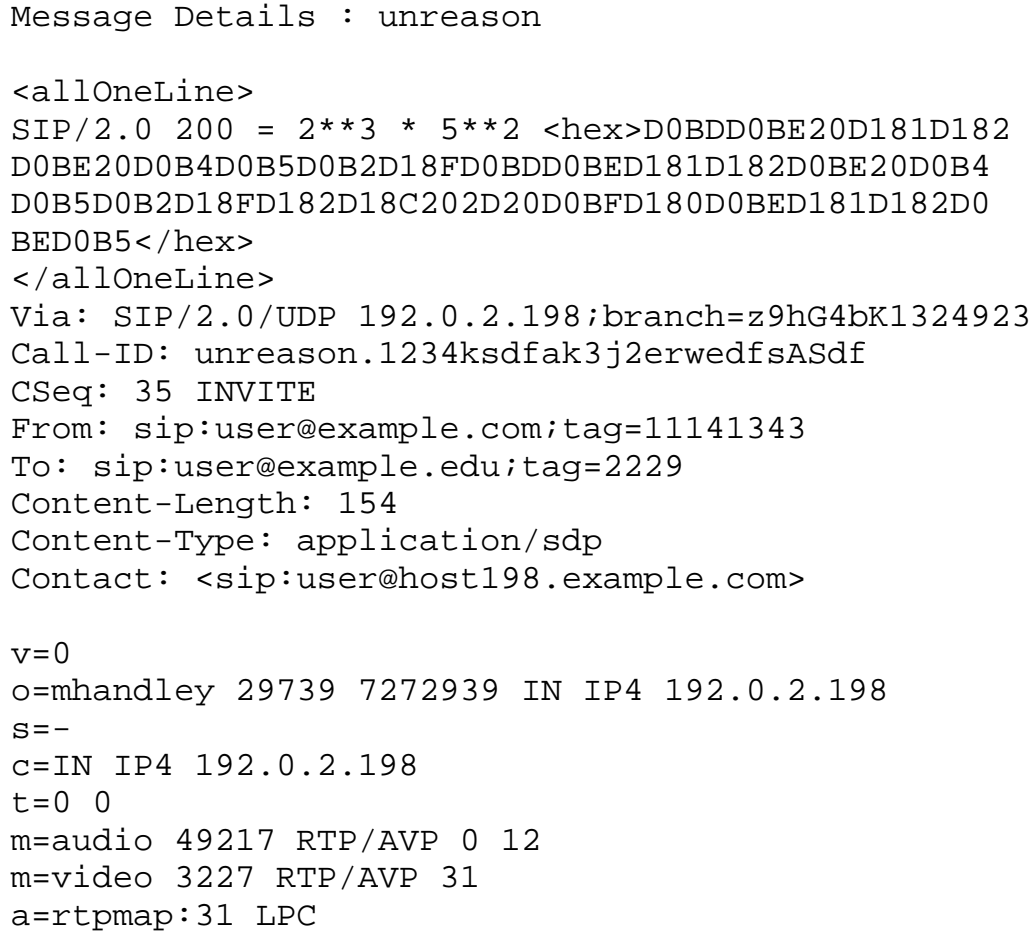

3.1.1.13. Empty Reason Phrase

This well-formed response contains no reason phrase. A parser must accept this message. The space character after the reason code is required. If it were not present, this message could be rejected as invalid (a liberal receiver would accept it anyway).

Message Details : noreason

$\operatorname{SIP} / 2.0100<$ hex $>20</$ hex $>$

Via: SIP/2.0/UDP 192.0.2.105; branch=z9hG4bK2398ndaoe

Call-ID: noreason.asndj203insdf99223ndf

CSeq: 35 INVITE

From: <sip:user@example.com>; tag=39ansfi3

To: <sip:user@example.edu>; tag=902 jndnke 3

Content-Length: 0

Contact: <sip:user@host105.example.com> 


\subsubsection{Invalid Messages}

This section contains several invalid messages reflecting errors seen at interoperability events and exploring important edge conditions that can be induced through malformed messages. This section does not attempt to be a comprehensive list of all types of invalid messages.

\subsubsection{Extraneous Header Field Separators}

The Via header field of this request contains additional semicolons and commas without parameters or values. The contact header field contains additional semicolons without parameters. This message is syntactically invalid.

An element receiving this request should respond with a 400 Bad Request error.

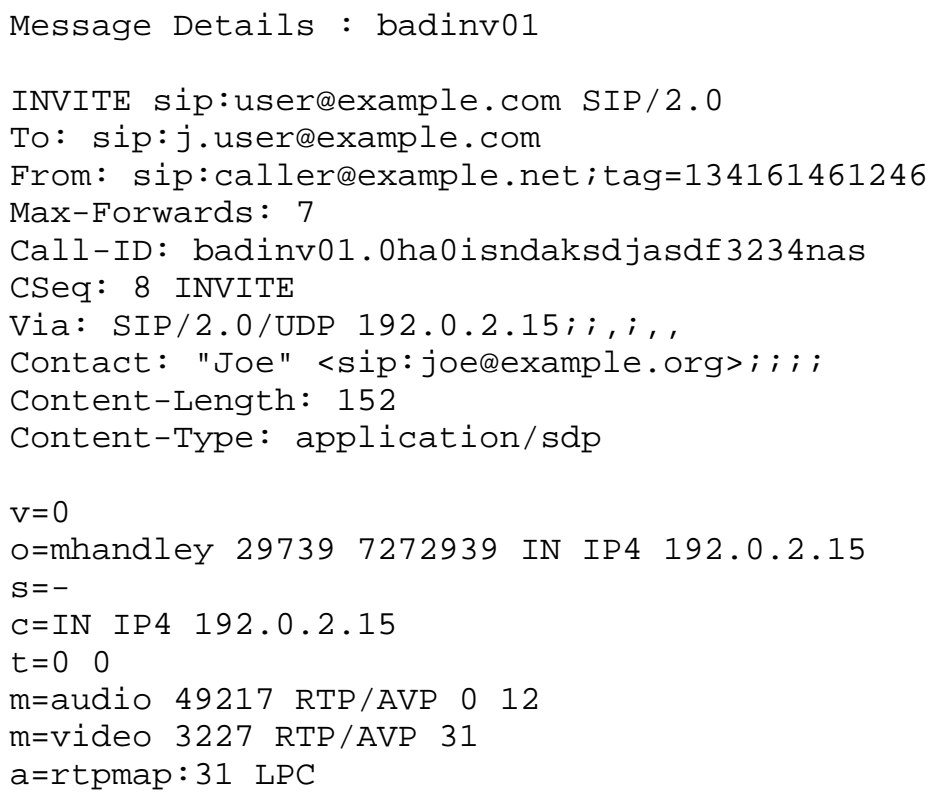

3.1.2.2. Content Length Larger Than Message

This is a request message with a content Length that is larger than the actual length of the body.

When sent over UDP (as this message ostensibly was), the receiving element should respond with a 400 Bad Request error. If this message arrived over a stream-based transport, such as TCP, there's not much 
the receiving party could do but wait for more data on the stream and close the connection if none is forthcoming within a reasonable period of time.

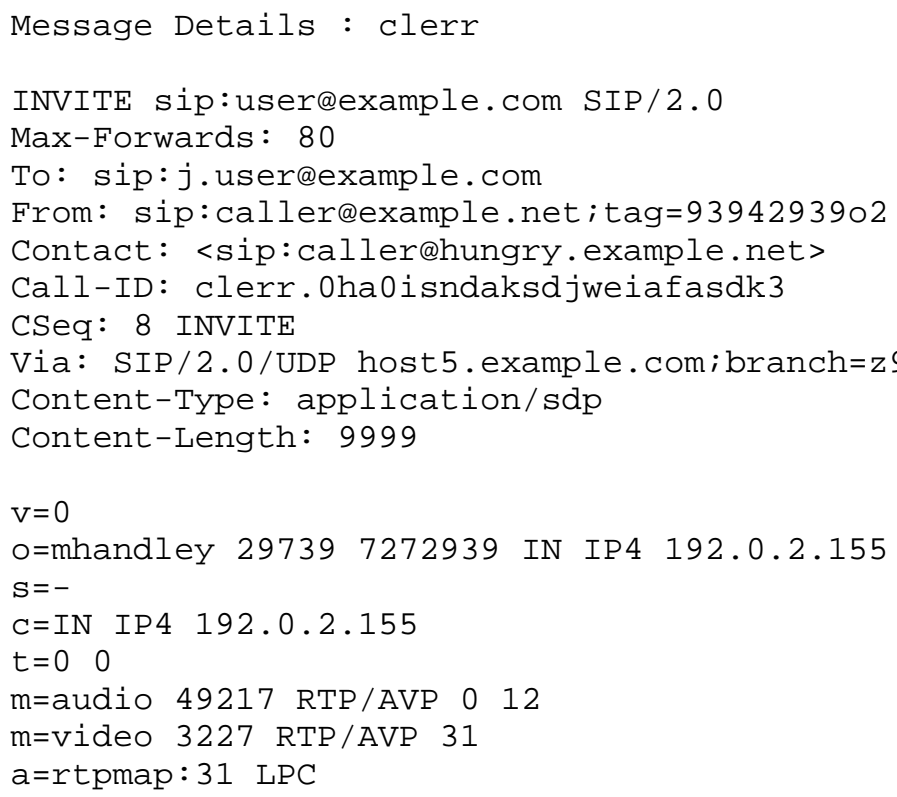

\subsubsection{Negative Content-Length}

This request has a negative value for Content-Length.

An element receiving this message should respond with an error. This request appeared over UDP, so the remainder of the datagram can simply be discarded. If a request like this arrives over TCP, the framing error is not recoverable, and the connection should be closed. The same behavior is appropriate for messages that arrive without a numeric value in the content-Length header field, such as the following:

\section{Content-Length: five}

Implementors should take extra precautions if the technique they choose for converting this ascii field into an integral form can return a negative value. In particular, the result must not be used as a counter or array index. 


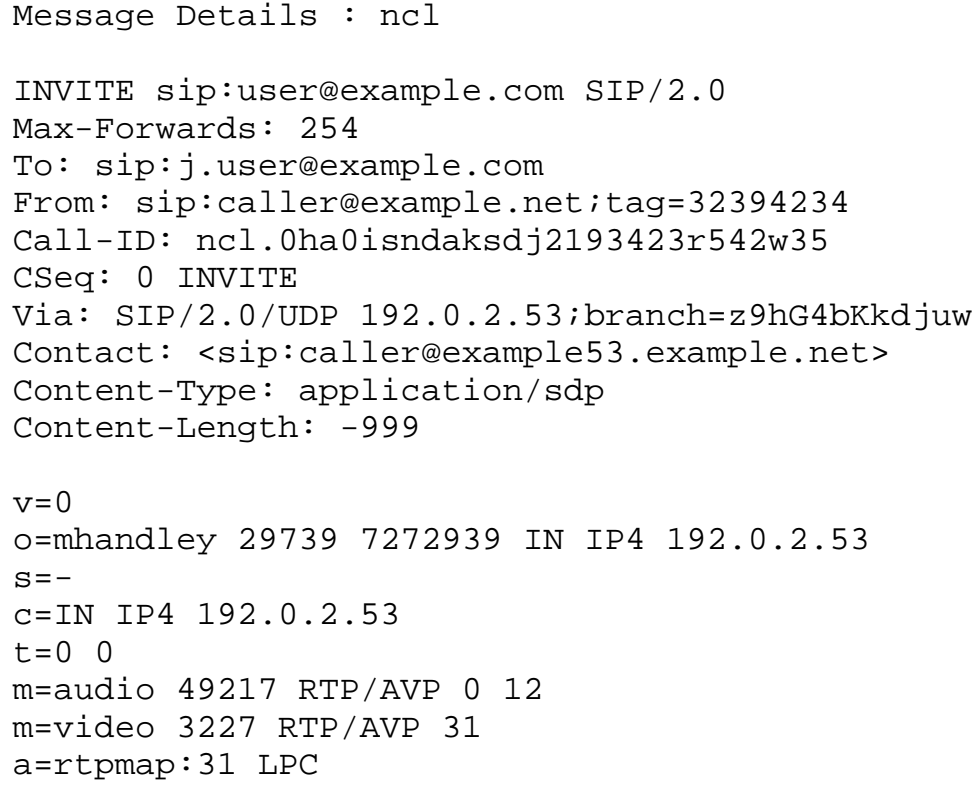

An element receiving this request should respond with a 400 Bad Request due to the CSeq error. If only the Max-Forwards field were in error, the element could choose to process the request as if the field were absent. If only the expiry values were in error, the element could treat them as if they contained the default values for expiration (3600 in this case).

Other scalar request fields that may contain aberrant values include, but are not limited to, the contact $q$ value, the Timestamp value, and the Via ttl parameter. 


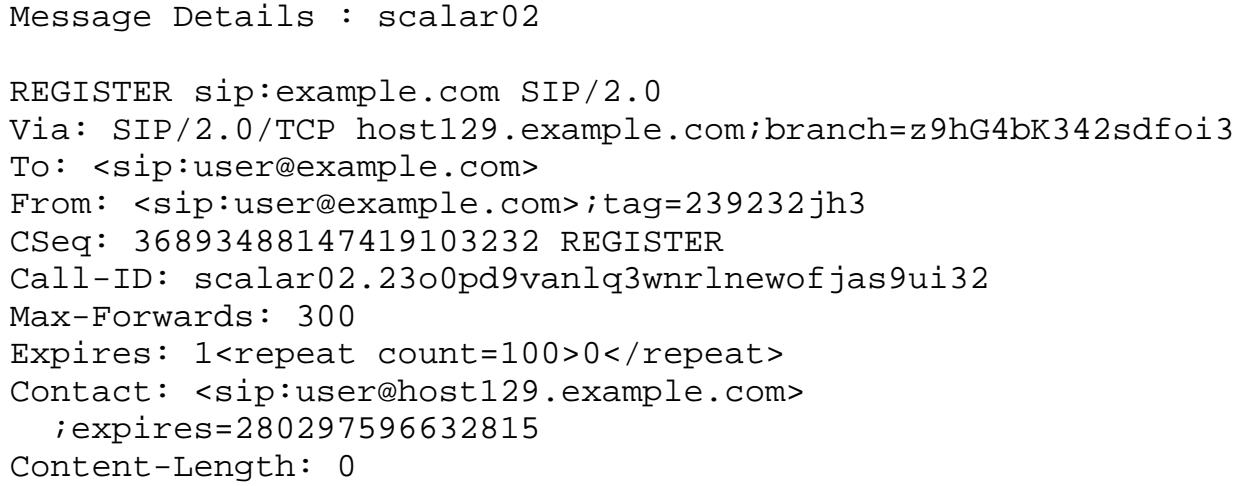

3.1.2.5. Response Scalar Fields with Overlarge Values

This response contains several scalar header field values outside their legal range.

- The CSeq sequence number is $>2 * \star 32-1$.

- The Retry-After field is unreasonably large (note that RFC 3261 does not define a legal range for this field).

- The Warning field has a warning-value with more than 3 digits.

An element receiving this response will simply discard it.

Message Details : scalarlg

SIP/2.0 503 Service Unavailable

<alloneLine>

Via: SIP/2.0/TCP host129.example.com

; branch=z9hG4bKzzxdiwo34sw

; received=192.0.2.129

$</ a l l o n e L i n e>$

To: <sip:userdexample.com>

From: <sip:otherdexample.net>; tag=2easdjfejw

CSeq: 9292394834772304023312 OPTIONS

Call-ID: scalarlg.noase 0 of $0234 \mathrm{hn} 2$ qofoaf 0232 aewf $2394 \mathrm{r}$

Retry-After: 949302838503028349304023988

Warning: 1812 overture "In Progress"

Content-Length: 0 


\subsubsection{Unterminated Quoted String in Display Name}

This is a request with an unterminated quote in the display name of the To field. An element receiving this request should return a 400 Bad Request error.

An element could attempt to infer a terminating quote and accept the message. Such an element needs to take care that it makes a reasonable inference when it encounters

To: "Mr J. User <sip:j.user@example.com> <sip:realj@example.net>

Message Details : quotbal

INVITE sip:user@example.com SIP/2.0

To: "Mr. J. User <sip:j.user@example.com>

From: sip:caller@example. net; tag $=93334$

Max-Forwards: 10

Call-ID: quotbal.aksdj

Contact: <sip:caller@host59.example.net>

CSeq: 8 INVITE

Via: SIP/2.0/UDP 192.0.2.59:5050; branch=z9hG4bKkdjuw39234

Content-Type: application/sdp

Content-Length: 152

$\mathrm{V}=0$

o=mhandley 297397272939 IN IP4 192.0 .2 .15

$\mathrm{S}=-$

$\mathrm{C}=\mathrm{IN}$ IP 4 192.0.2.15

$t=0 \quad 0$

m=audio 49217 RTP/AVP 012

$\mathrm{m}=\mathrm{video} 3227 \mathrm{RTP} / \mathrm{AVP} 31$

a=rtpmap: 31 LPC 


\subsubsection{7. <> Enclosing Request-URI}

This INVITE request is invalid because the Request-URI has been enclosed within in "<>".

It is reasonable always to reject a request with this error with a 400 Bad Request. Elements attempting to be liberal with what they accept may choose to ignore the brackets. If the element forwards the request, it must not include the brackets in the messages it sends.

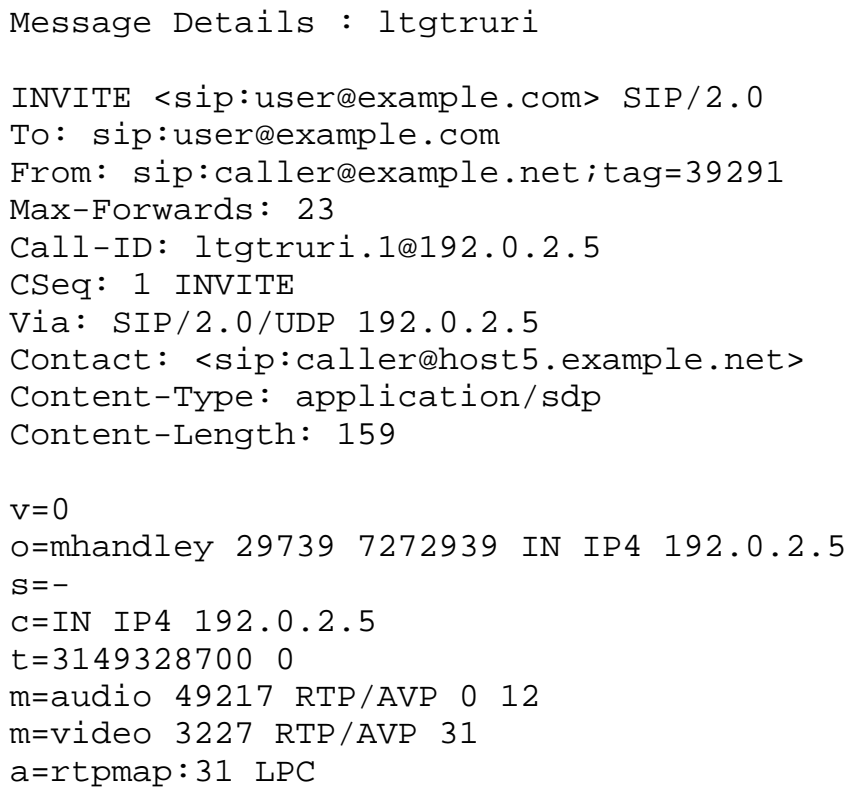




\subsubsection{Malformed SIP Request-URI (embedded LWS)}

This INVITE has illegal LWS within the Request-URI.

An element receiving this request should respond with a 400 Bad Request.

An element could attempt to ignore the embedded LWS for those schemes (like SIP) where doing so would not introduce ambiguity.

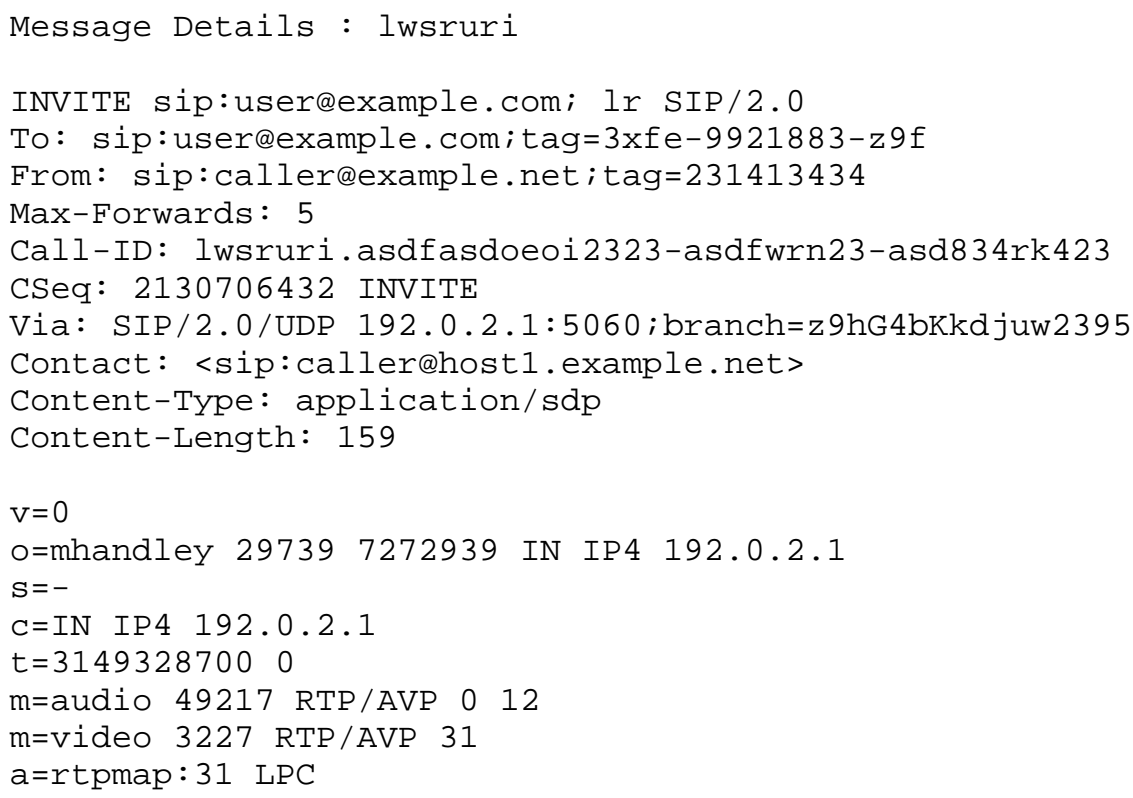




\subsubsection{Multiple SP Separating Request-Line Elements}

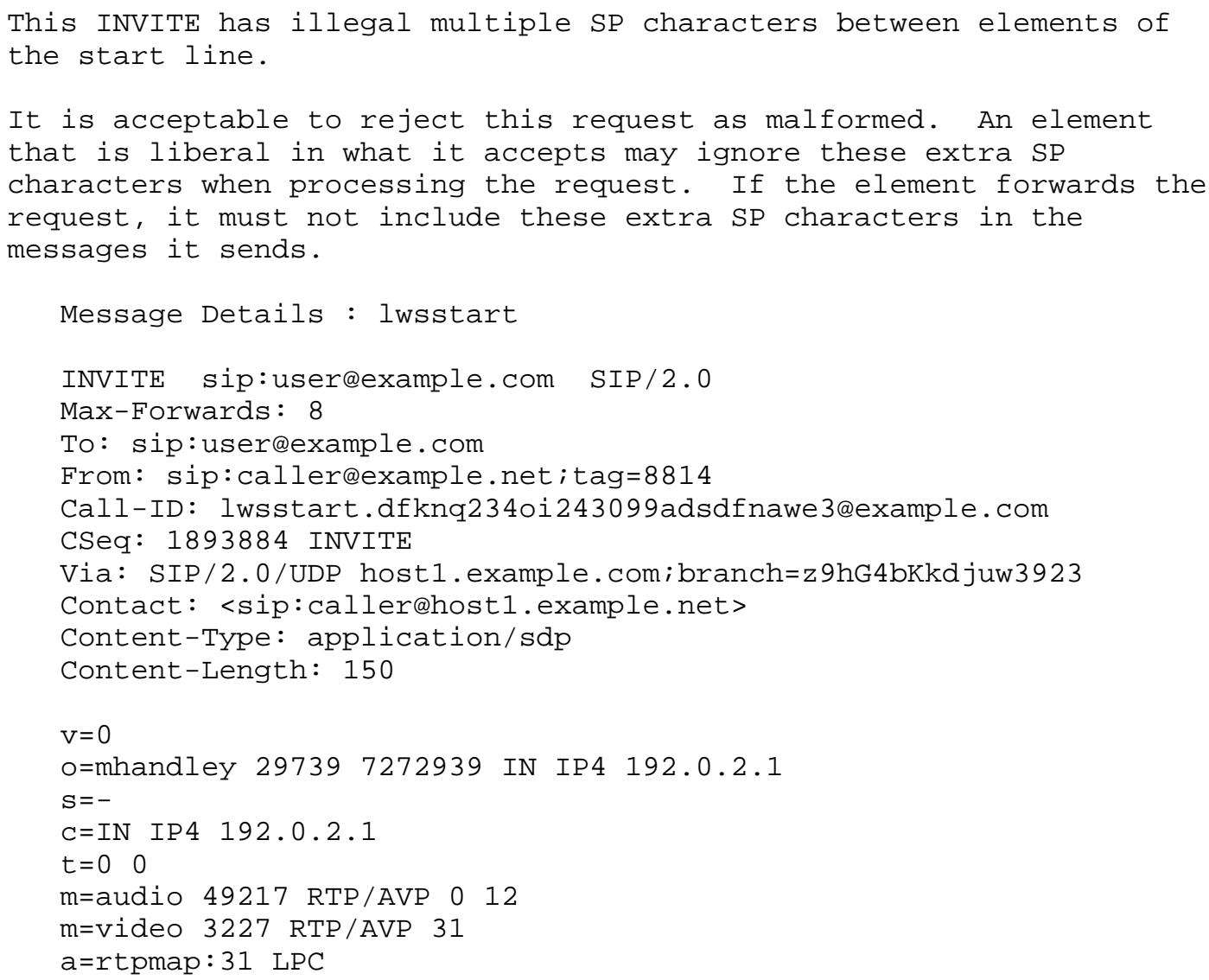




\subsubsection{SP Characters at End of Request-Line}

This OPTIONS request contains SP characters between the SIP-Version field and the CRLF terminating the Request-Line.

It is acceptable to reject this request as malformed. An element that is liberal in what it accepts may ignore these extra SP characters when processing the request. If the element forwards the request, it must not include these extra SP characters in the messages it sends.

Message Details : trws

OPTIONS sip:remote-targeteexample.com SIP $/ 2.0<$ hex $>2020</$ hex $>$

Via: SIP/2.0/TCP host1.example.com;branch=z9hG4bK299342093

To: <sip:remote-target@example.com>

From: <sip:local-resourcedexample.com>; tag=329429089

Call-ID: trws.oicu34958239neffasdhr2345r

Accept: application/sdp

CSeq: 238923 OPTIONS

Max-Forwards: 70

Content-Length: 0 


\subsubsection{Escaped Headers in SIP Request-URI}

This INVITE is malformed, as the SIP Request-URI contains escaped headers.

It is acceptable for an element to reject this request with a $400 \mathrm{Bad}$ Request. An element could choose to be liberal in what it accepts and ignore the escaped headers. If the element is a proxy, the escaped headers must not appear in the Request-URI of the forwarded request (and most certainly must not be translated into the actual header of the forwarded request).

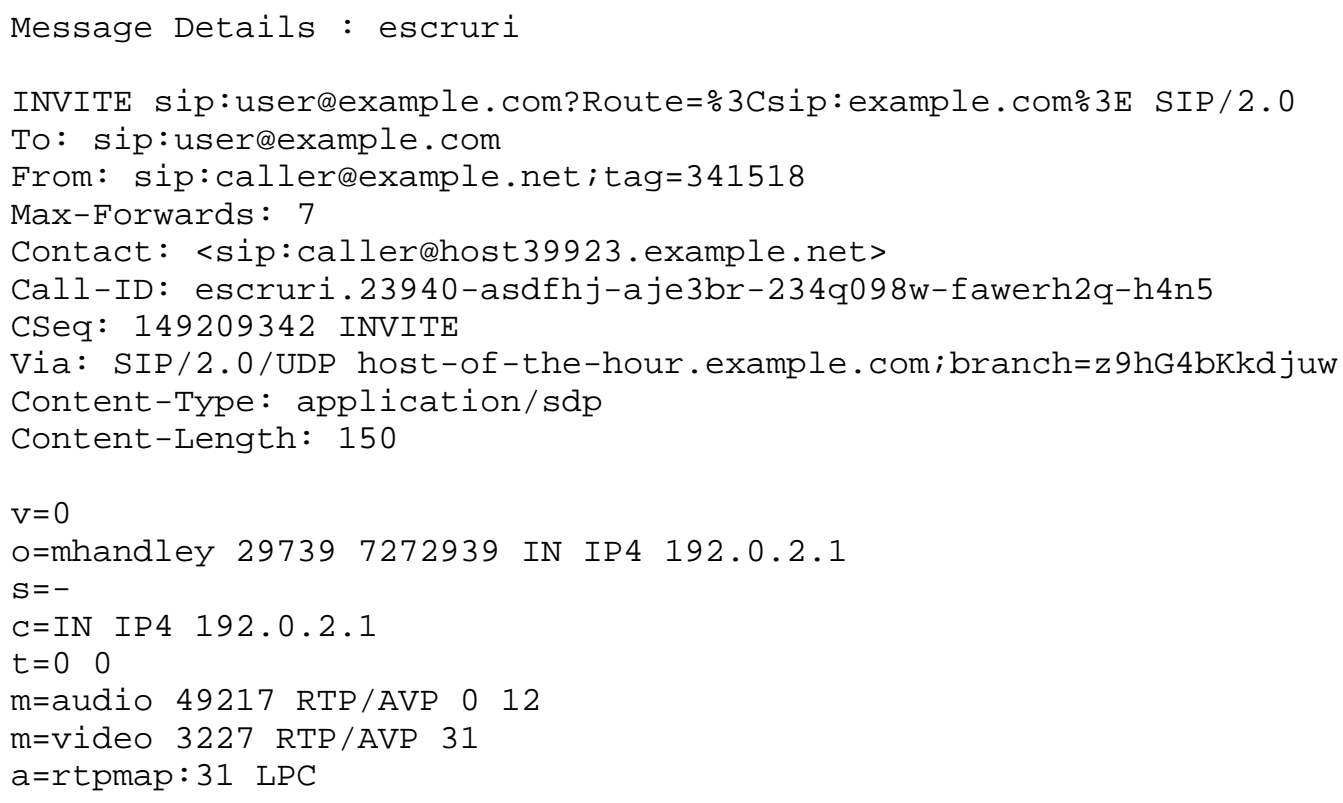




\subsubsection{Invalid Time Zone in Date Header Field}

This INVITE is invalid, as it contains a non-GMT time zone in the SIP Date header field.

It is acceptable to reject this request as malformed (though an element shouldn't do that unless the contents of the Date header field were actually important to its processing). An element wishing to be liberal in what it accepts could ignore this value altogether if it wasn't going to use the Date header field anyway. Otherwise, it could attempt to interpret this date and adjust it to GMT.

RFC 3261 explicitly defines the only acceptable time zone designation as "GMT". "UT", while synonymous with GMT per [RFC2822], is not valid. "UTC" and "UCT" are also invalid.

Message Details : baddate

INVITE sip:user@example.com SIP/2.0

To: sip:user@example.com

From: sip: caller@example. net; tag $=2234923$

Max-Forwards: 70

Call-ID: baddate.239423mnsadf3j23lj42--sedfnm234

CSeq: 1392934 INVITE

Via: SIP/2.0/UDP host.example.com; branch=z9hG4bKkdjuw

Date: Fri, 01 Jan 2010 16:00:00 EST

Contact: <sip:caller@host5.example.net>

Content-Type: application/sdp

Content-Length: 150

$\mathrm{V}=0$

o=mhandley 297397272939 IN IP4 192.0.2.5

$\mathrm{S}=-$

$\mathrm{C}=\mathrm{IN}$ IP 4 192.0.2.5

$t=0 \quad 0$

m=audio 49217 RTP/AVP 012

$\mathrm{m}=\mathrm{video} 3227$ RTP/AVP 31

$\mathrm{a}=$ rtpmap: 31 LPC 


\subsubsection{Failure to Enclose name-addr URI in <>}

This REGISTER request is malformed. The SIP URI contained in the Contact Header field has an escaped header, so the field must be in name-addr form (which implies that the URI must be enclosed in <>).

It is reasonable for an element receiving this request to respond with a 400 Bad Request. An element choosing to be liberal in what it accepts could infer the angle brackets since there is no ambiguity in this example. In general, that won't be possible.

Message Details : regbadct

REGISTER sip:example.com SIP/2.0

To: sip:user@example.com

From: sip:userdexample.com; tag $=998332$

Max-Forwards: 70

Call-ID: regbadct.k345asrl3fdbve10.0.0.1

CSeq: 1 REGISTER

Via: SIP/2.0/UDP 135.180.130.133:5060; branch=z9hG4bKkdjuw

Contact: sip:user@example.com?Route=\%3Csip:sip.example.com\%3E

$1: 0$

3.1.2.14. Spaces within addr-spec

This request is malformed, since the addr-spec in the To header field contains spaces. Parsers receiving this request must not break. It is reasonable to reject this request with a 400 Bad Request response. Elements attempting to be liberal may ignore the spaces.

Message Details : badaspec

OPTIONS sip:user@example.org SIP/2.0

Via: SIP/2.0/UDP host4.example.com:5060; branch=z9hG4bKkdju43234

Max-Forwards: 70

From: "Bell, Alexander" <sip:a.g.bell@example.com>; tag=433423

To: "Watson, Thomas" < sip:t.watson@example.org >

Call-ID: badaspec.sdf0234n2nds0a099u23h3hnnw009cdkne3

Accept: application/sdp

CSeq: 3923239 OPTIONS

$1: 0$ 


\subsubsection{Non-token Characters in Display Name}

This OPTIONS request is malformed, since the display names in the To and From header fields contain non-token characters but are unquoted.

It is reasonable always to reject this kind of error with a 400 Bad Request response.

An element may attempt to be liberal in what it receives and infer the missing quotes. If this element were a proxy, it must not propagate the error into the request it forwards. As a consequence, if the fields are covered by a signature, there's not much point in trying to be liberal - the message should simply be rejected.

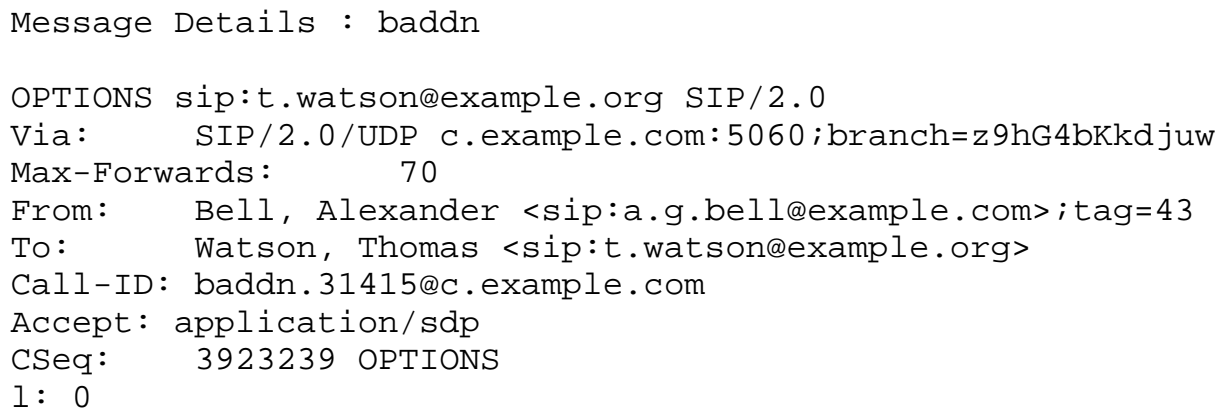

\subsubsection{Unknown Protocol Version}

To an element implementing [RFC3261], this request is malformed due to its high version number.

The element should respond to the request with a 505 Version Not Supported error.

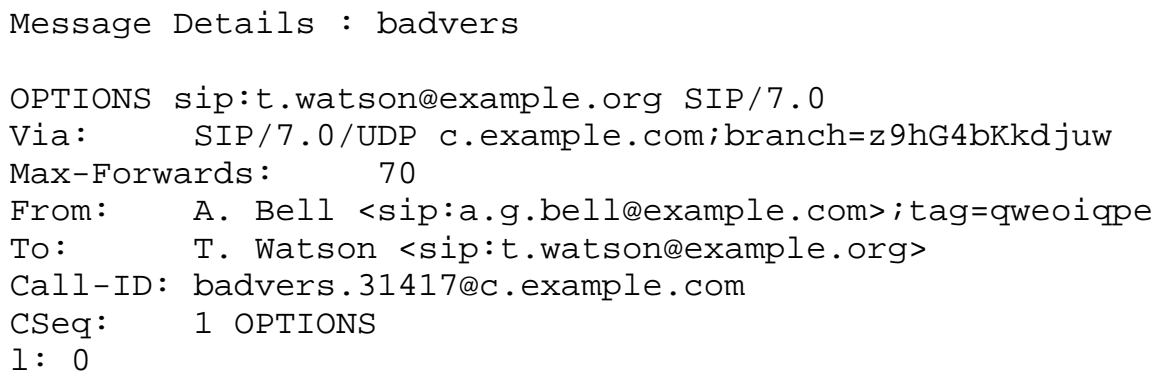




\subsubsection{Start Line and CSeq Method Mismatch}

This request has mismatching values for the method in the start line and the cSeq header field. Any element receiving this request will respond with a 400 Bad Request.

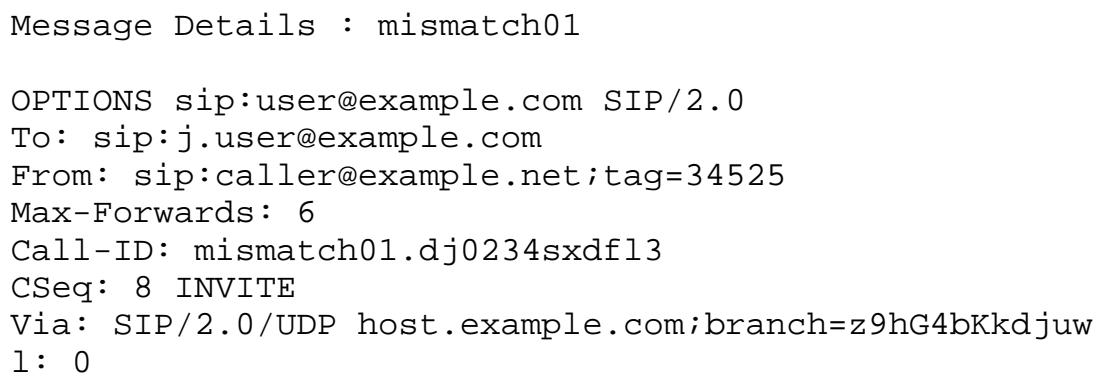

\subsubsection{Unknown Method with CSeq Method Mismatch}

This message has an unknown method in the start line, and a CSeq method tag that does not match.

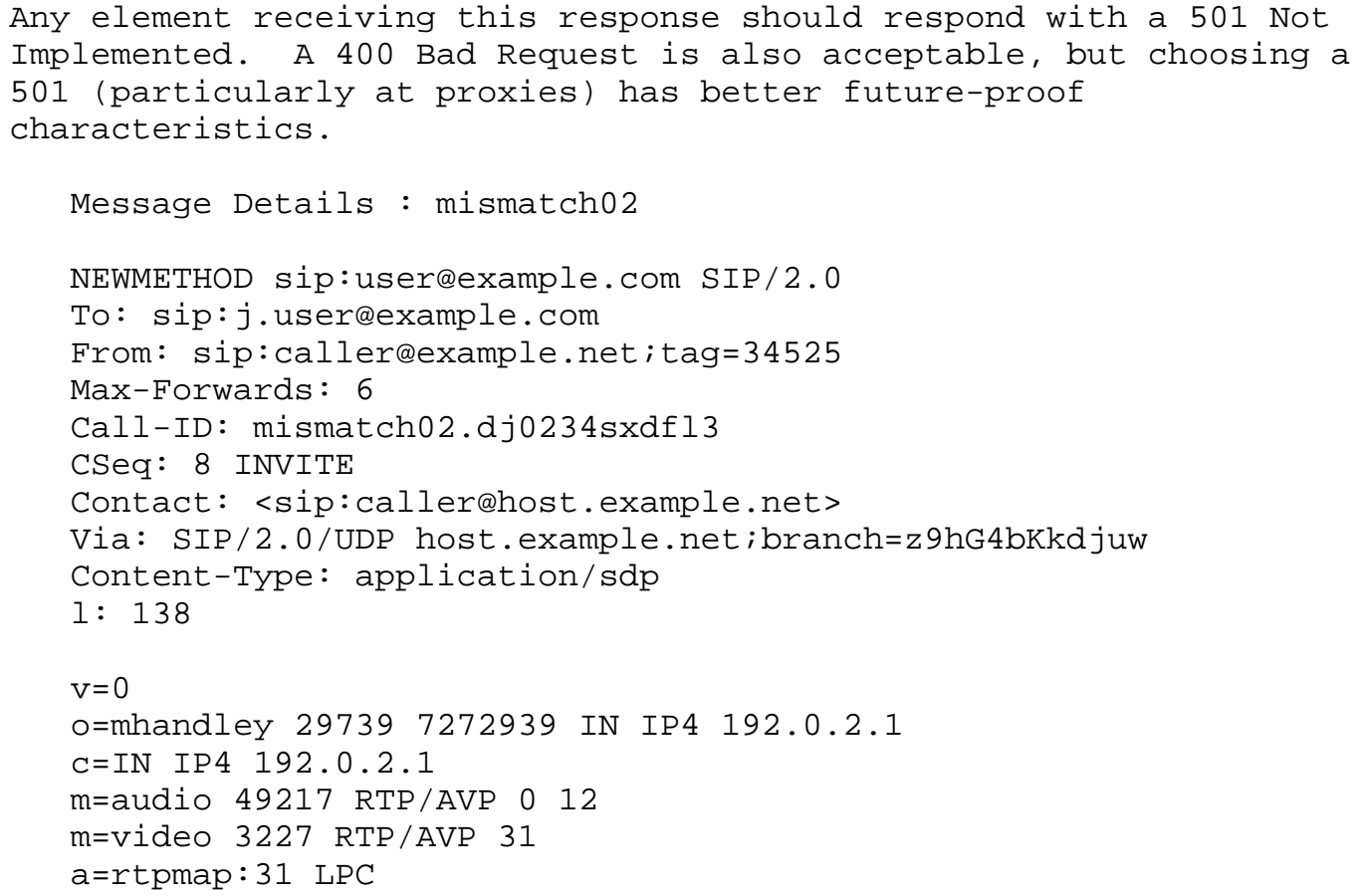




\subsubsection{Overlarge Response Code}

This response has a response code larger than 699. An element receiving this response should simply drop it.

Message Details : bigcode

SIP/2.0 4294967301 better not break the receiver

Via: SIP/2.0/UDP 192.0.2.105; branch=z9hG4bK2398ndaoe

Call-ID: bigcode.asdof3uj203asdnf3429uasdhfas3ehjasdfas $9 i$

CSeq: 353494 INVITE

From: <sip:user@example.com>; tag=39ansfi3

To: <sip:user@example.edu>; tag=902 jndnke3

Content-Length: 0

Contact: <sip:user@host105.example.com>

\subsection{Transaction Layer Semantics}

This section contains tests that exercise an implementation's parser and transaction-layer logic.

\subsubsection{Missing Transaction Identifier}

This request indicates support for RFC 3261-style transaction identifiers by providing the $z 9 h \mathrm{~h} 4 \mathrm{bK}$ prefix to the branch parameter, but it provides no identifier. A parser must not break when receiving this message. An element receiving this request could reject the request with a 400 Response (preferably statelessly, as other requests from the source are likely also to have a malformed branch parameter), or it could fall back to the RFC 2543-style transaction identifier.

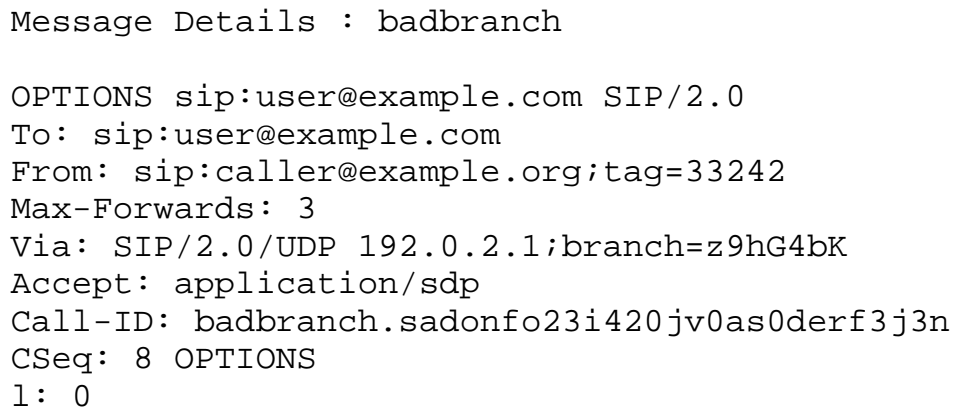




\subsection{Application-Layer Semantics}

This section contains tests that exercise an implementation's parser and application-layer logic.

3.3.1. Missing Required Header Fields

This request contains no Call-ID, From, or To header fields.

An element receiving this message must not break because of the missing information. Ideally, it will respond with a 400 Bad Request error.

Message Details : insuf

INVITE sip:userdexample.com SIP/2.0

CSeq: 193942 INVITE

Via: SIP/2.0/UDP 192.0.2.95; branch=z9hG4bKkdj.insuf

Content-Type: application/sdp

1: 152

$\mathrm{V}=0$

o=mhandley 297397272939 IN IP4 192.0.2.95

$\mathrm{S}=-$

$\mathrm{C}=\mathrm{IN}$ IP $4 \quad 192.0 .2 .95$

$t=0 \quad 0$

m=audio 49217 RTP/AVP 012

$\mathrm{m}=\mathrm{video} 3227$ RTP/AVP 31

a=rtpmap:31 LPC 


\subsubsection{Request-URI with Unknown Scheme}

This OPTIONS contains an unknown URI scheme in the Request-URI. A parser must accept this as a well-formed SIP request.

An element receiving this request will reject it with a 416 Unsupported URI Scheme response.

Some early implementations attempt to look at the contents of the To header field to determine how to route this kind of request. That is an error. Despite the fact that the To header field and the Request URI frequently look alike in simplistic first-hop messages, the To header field contains no routing information.

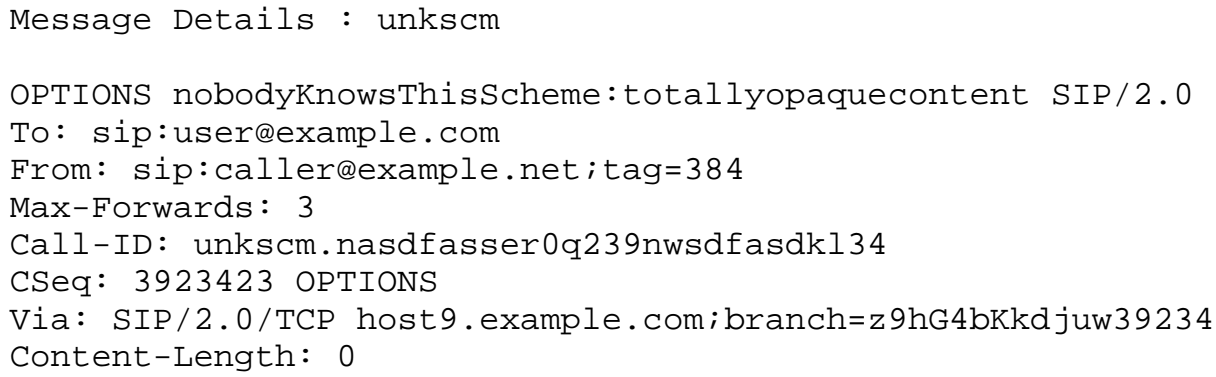

\subsubsection{Request-URI with Known but Atypical Scheme}

This OPTIONS contains an Request-URI with an IANA-registered scheme that does not commonly appear in Request-URIs of SIP requests. A parser must accept this as a well-formed SIP request.

If an element will never accept this scheme as meaningful in a Request-URI, it is appropriate to treat it as unknown and return a 416 Unsupported URI Scheme response. If the element might accept some URIs with this scheme, then a 404 Not Found is appropriate for those URIs it doesn't accept.

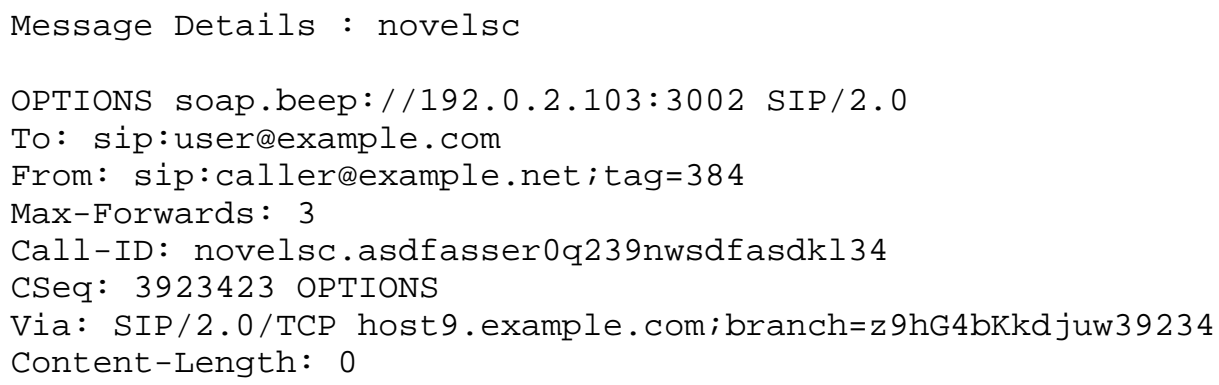




\subsubsection{Unknown URI Schemes in Header Fields}

This message contains registered schemes in the To, From, and Contact header fields of a request. The message is syntactically valid. Parsers must not fail when receiving this message.

Proxies should treat this message as they would any other request for this URI. A registrar would reject this request with a $400 \mathrm{Bad}$

Request response, since the To: header field is required to contain a SIP or SIPS URI as an AOR.

Message Details : unksm2

REGISTER sip:example.com SIP/2.0

To: isbn:2983792873

From: <http://www.example.com>; tag=3234233

Call-ID: unksm2.daksdj@hyphenated-host.example.com

CSeq: 234902 REGISTER

Max-Forwards: 70

Via: SIP/2.0/UDP 192.0.2.21:5060; branch=z9hG4bKkdjuw

Contact: <name:John_Smith>

$1: 0$

3.3.5. Proxy-Require and Require

This request tests proper implementation of SIP'S Proxy-Require and Require extension mechanisms.

Any element receiving this request will respond with a 420 Bad Extension response, containing an Unsupported header field listing these features from either the Require or Proxy-Require header field, depending on the role in which the element is responding.

Message Details : bext01

OPTIONS sip:user@example.com SIP/2.0

To: sip:j_user@example.com

From: sip:caller@example.net; tag=242etr

Max-Forwards: 6

Call-ID: bext01.0ha0isndaksdj

Require: nothingSupportsThis, nothingSupportsThisEither

Proxy-Require: noProxiesSupportThis, norDoAnyProxiessupportThis CSeq: 8 OPTIONS

Via: SIP/2.0/TLS fold-and-staple.example.com;branch=z9hG4bkkdjuw Content-Length: 0 


\subsubsection{Unknown Content-Type}

This INVITE request contains a body of unknown type. It is syntactically valid. A parser must not fail when receiving it.

A proxy receiving this request would process it just as it would any other INVITE. An endpoint receiving this request would reject it with a 415 Unsupported Media Type error.

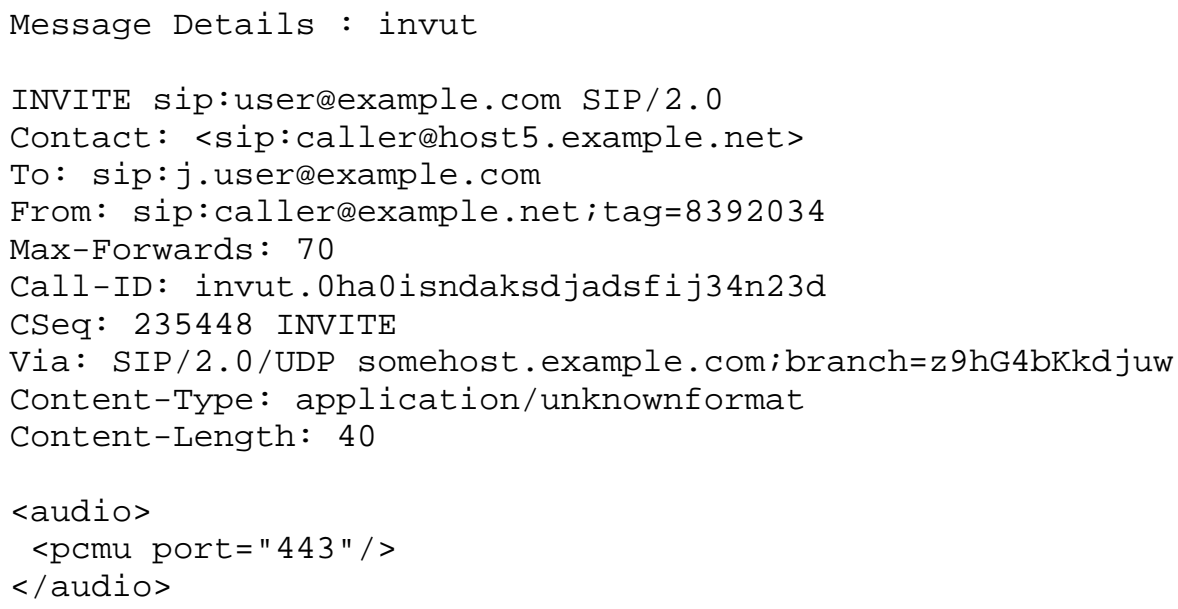

\subsubsection{Unknown Authorization Scheme}

This REGISTER request contains an Authorization header field with an unknown scheme. The request is well formed. A parser must not fail when receiving it.

A proxy will treat this request as it would any other REGISTER. If it forwards the request, it will include this Authorization header field unmodified in the forwarded messages.

A registrar that does not care about challenge-response authentication will simply ignore the Authorization header field, processing this registration as if the field were not present. A registrar that does care about challenge-response authentication will reject this request with a 401 , issuing a new challenge with a scheme it understands.

Endpoints choosing not to act as registrars will simply reject the request. A 405 Method Not Allowed is appropriate. 
Message Details : regaut01

REGISTER sip:example.com SIP/2.0

To: sip:j.userdexample.com

From: sip:j.user@example.com;tag=87321hj23128

Max-Forwards: 8

Call-ID: regaut01.0ha0isndaksdj

CSeq: 9338 REGISTER

Via: SIP/2.0/TCP 192.0.2.253; branch=z9hG4bKkdjuw

Authorization: NooneKnowsThisScheme opaque-data=here

Content-Length: 0

\subsubsection{Multiple Values in Single Value Required Fields}

The message contains a request with multiple Call-ID, To, From, MaxForwards, and CSeq values. An element receiving this request must not break.

An element receiving this request would respond with a 400 Bad Request error.

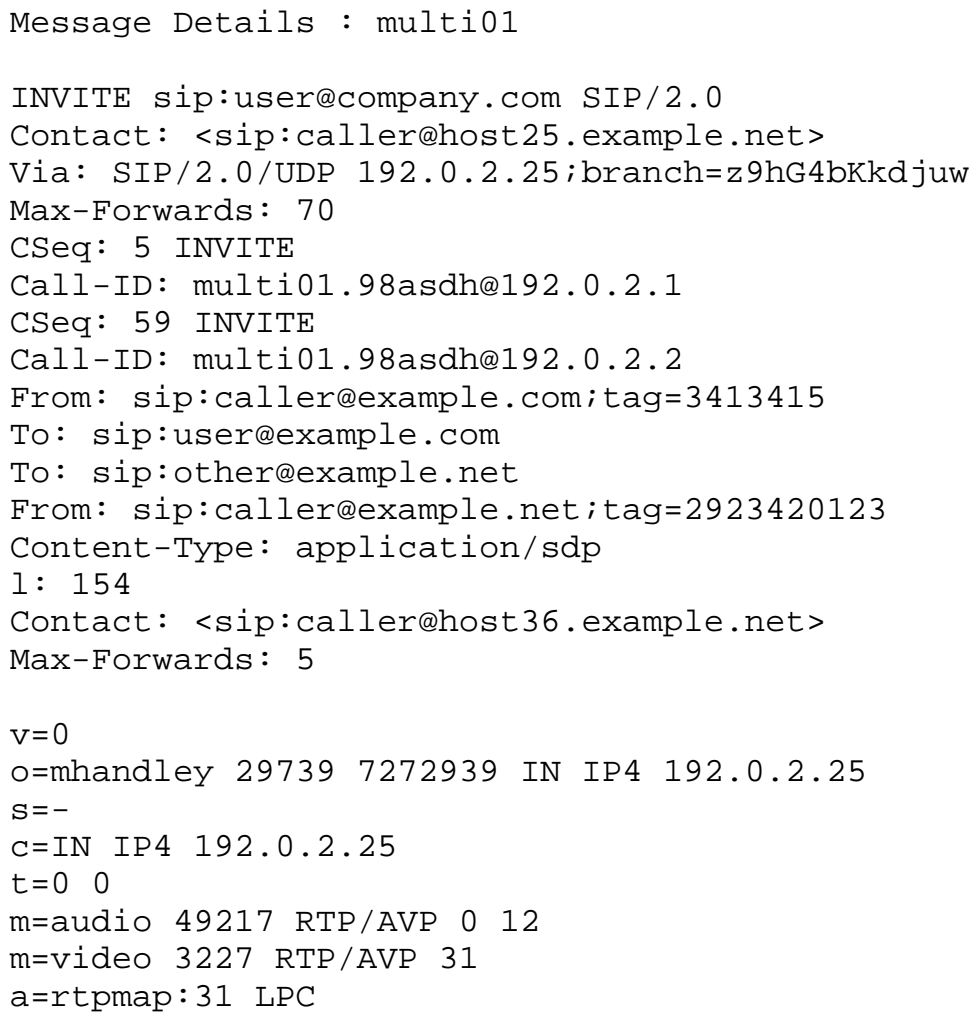




\subsubsection{Multiple Content-Length Values}

Multiple conflicting Content-Length header field values appear in this request.

From a framing perspective, this situation is equivalent to an invalid Content-Length value (or no value at all).

An element receiving this message should respond with an error. This request appeared over UDP, so the remainder of the datagram can simply be discarded. If a request like this arrives over TCP, the framing error is not recoverable, and the connection should be closed.

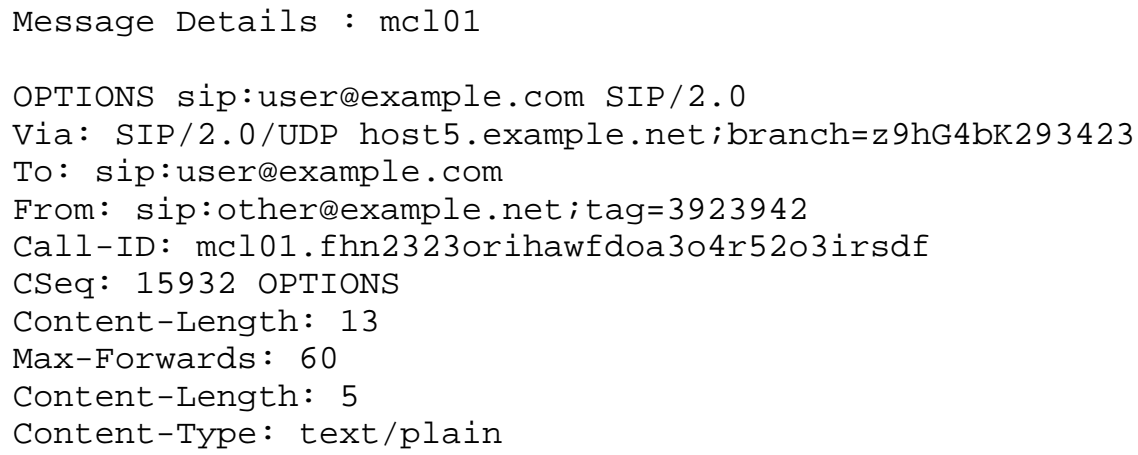

There's no way to know how many octets are supposed to be here.

\subsubsection{OK Response with Broadcast Via Header Field Value}

This message is a response with a 2 nd Via header field value's sentby containing 255.255.255.255. The message is well formed; parsers must not fail when receiving it.

Per [RFC3261], an endpoint receiving this message should simply discard it.

If a proxy followed normal response processing rules blindly, it would forward this response to the broadcast address. To protect against this as an avenue of attack, proxies should drop such responses. 


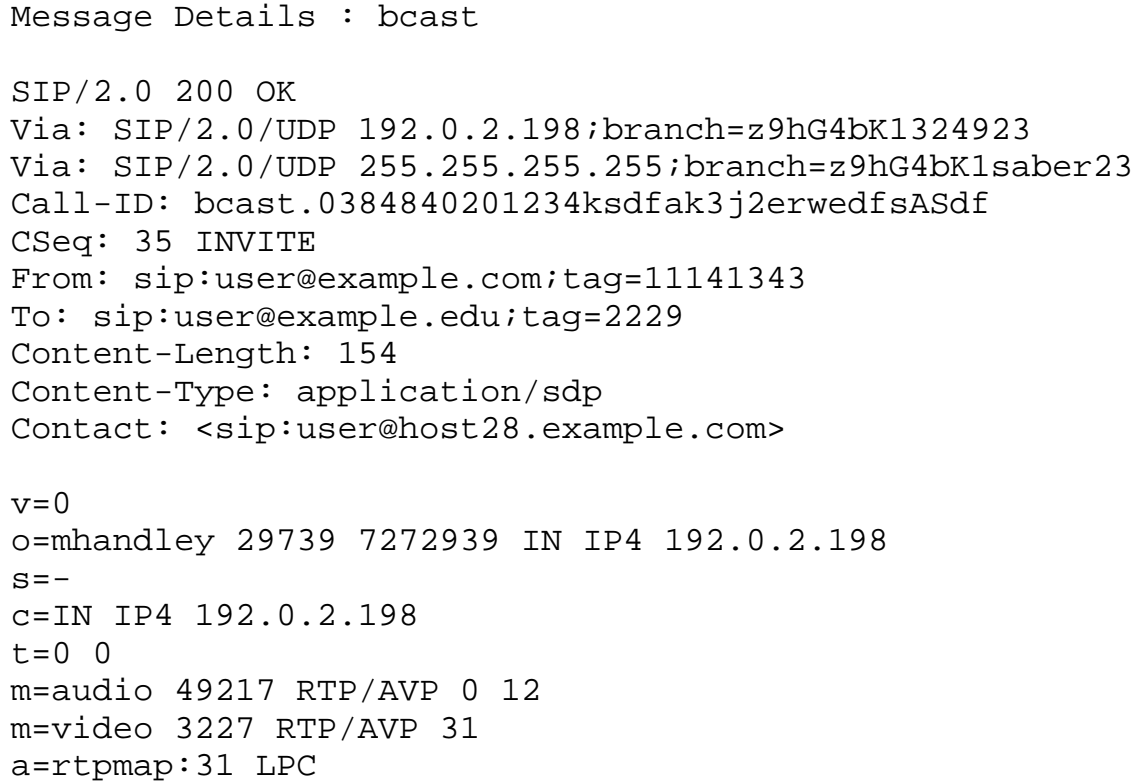

3.3.11. Max-Forwards of Zero

This is a legal SIP request with the Max-Forwards header field value set to zero.

A proxy should not forward the request and should respond 483 (Too Many Hops). An endpoint should process the request as if the MaxForwards field value were still positive.

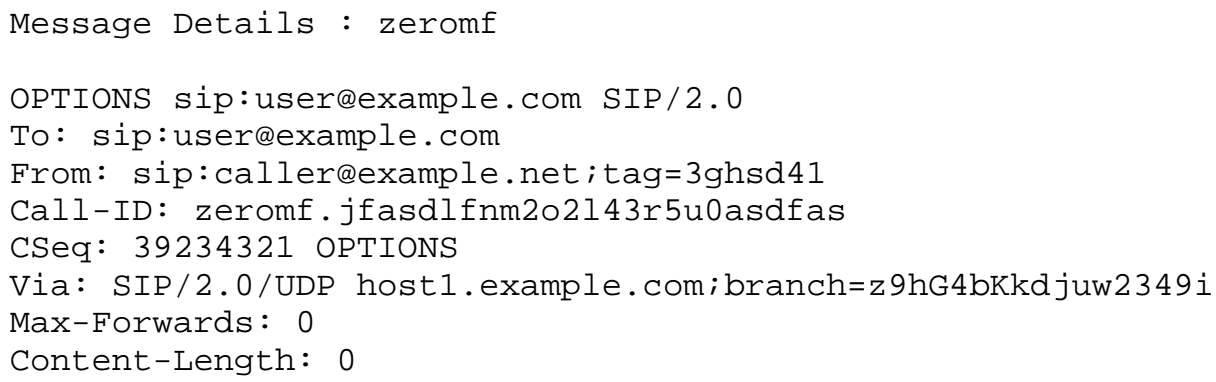




\subsubsection{REGISTER with a Contact Header Parameter}

This register request contains a contact where the 'unknownparam' parameter must be interpreted as a contact-param and not a url-param.

This REGISTER should succeed. The response must not include "unknownparam" as a url-parameter for this binding. Likewise, "unknownparam" must not appear as a url-parameter in any binding during subsequent fetches.

Behavior is the same, of course, for any known contact-param parameter names.

Message Details : cparam01

REGISTER sip:example.com SIP/2.0

Via: SIP/2.0/UDP saturn.example.com:5060; branch=z9hG4bKkdjuw Max-Forwards: 70

From: sip:watson@example.com; tag=DkfVgjkrtMwaerkKpe

To: sip:watson@example.com

Call-ID: cparam01.70710@saturn.example.com

CSeq: 2 REGISTER

Contact: sip:+19725552222@gw1.example.net; unknownparam

$1: 0$

\subsubsection{REGISTER with a url-parameter}

This register request contains a contact where the URI has an unknown parameter.

The register should succeed, and a subsequent retrieval of the registration must include "unknownparam" as a url-parameter.

Behavior is the same, of course, for any known url-parameter names.

Message Details : cparam02

REGISTER sip:example.com SIP/2.0

Via: SIP/2.0/UDP saturn.example.com:5060; branch=z9hG4bKkdjuw

Max-Forwards: 70

From: sip: watson@example.com; tag $=838293$

To: sip:watson@example.com

Call-ID: cparam02.70710@saturn.example.com

CSeq: 3 REGISTER

Contact: <sip:+19725552222@gw1.example.net; unknownparam> $1: 0$ 


\subsubsection{REGISTER with a URL Escaped Header}

This register request contains a contact where the URI has an escaped header.

The register should succeed, and a subsequent retrieval of the registration must include the escaped Route header in the contact URI for this binding.

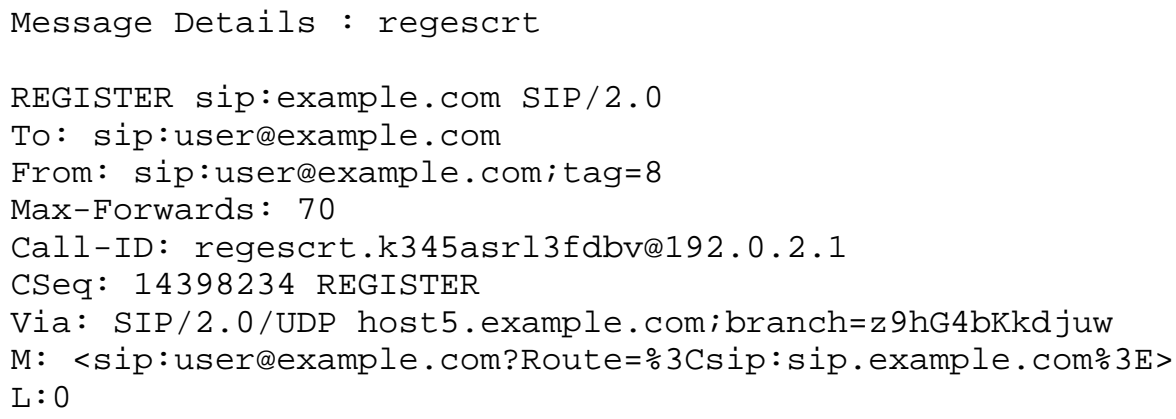




\subsubsection{Unacceptable Accept Offering}

This request indicates that the response must contain a body in an unknown type. In particular, since the Accept header field does not contain application/sdp, the response may not contain an SDP body. The recipient of this request could respond with a 406 Not Acceptable, with a Warning/399 indicating that a response cannot be formulated in the formats offered in the Accept header field. It is also appropriate to respond with a 400 Bad Request, since all SIP User-Agents (UAs) supporting INVITE are required to support application/sdp.

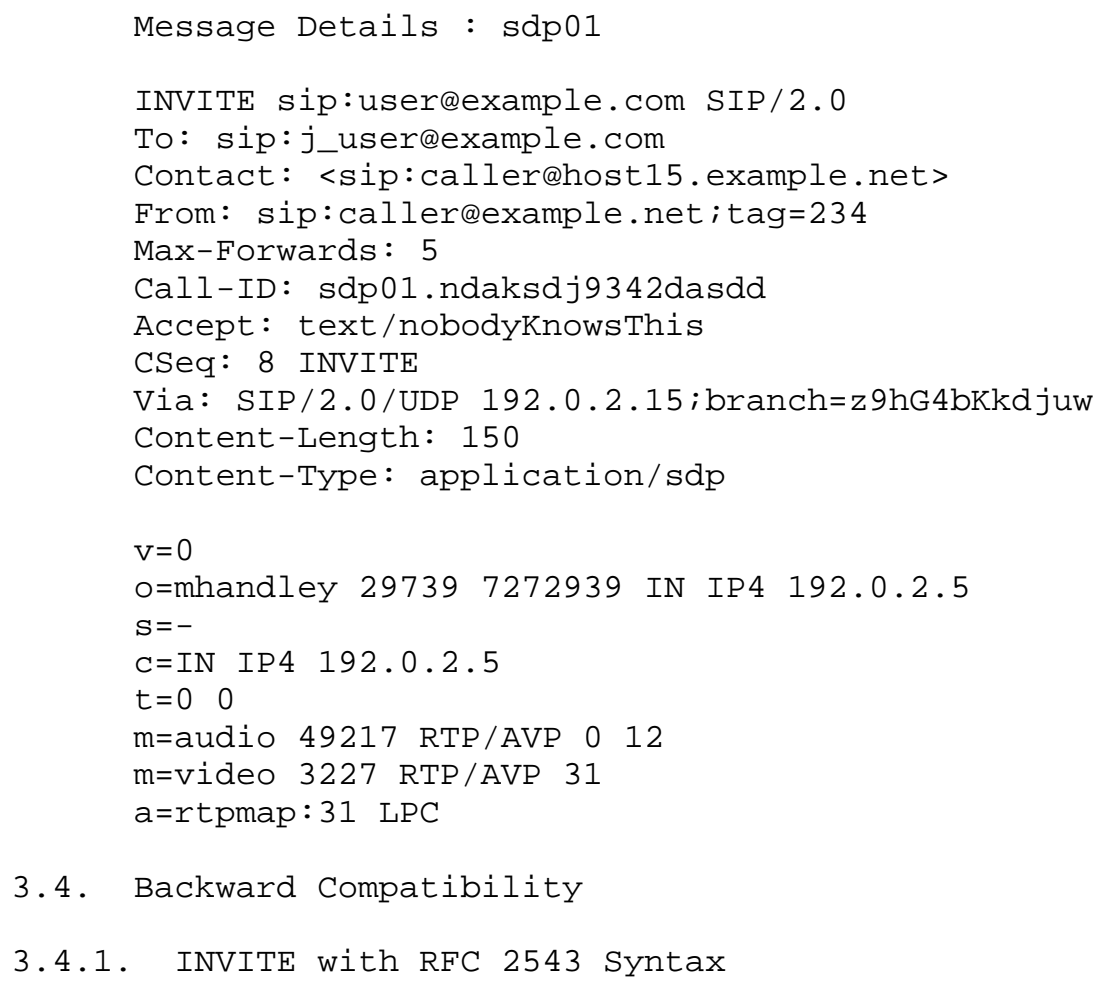

3.4. Backward Compatibility

\subsubsection{INVITE with RFC 2543 syntax}

This is a legal message per RFC 2543 (and several bis versions) that should be accepted by RFC 3261 elements that want to maintain backwards compatibility.

- There is no branch parameter at all on the Via header field value.

o There is no From tag. 
- There is no explicit Content-Length. (The body is assumed to be all octets in the datagram after the null-line.)

o There is no Max-Forwards header field.

Message Details : inv2543

INVITE sip:UserB@example.com SIP/2.0

Via: SIP/2.0/UDP iftgw.example.com

From: <sip:+13035551111@ift.client.example.net; user=phone>

Record-Route: <sip:Userblexample.com; maddr=ssl.example.com>

To: sip:+16505552222@ss1.example.net; user=phone

Call-ID: inv2543.1717@ift.client.example.com

CSeq: 56 INVITE

Content-Type: application/sdp

$\mathrm{V}=0$

o=mhandley 297397272939 IN IP4 192.0.2.5

$\mathrm{S}=-$

$\mathrm{C}=\mathrm{IN}$ IP 4 192.0.2.5

$t=0 \quad 0$

m=audio 49217 RTP/AVP 0

4. Security Considerations

This document presents NON-NORMATIVE examples of SIP session establishment. The security considerations in [RFC3261] apply.

Parsers must carefully consider edge conditions and malicious input as part of their design. Attacks on many Internet systems use crafted input to cause implementations to behave in undesirable ways. Many of the messages in this document are designed to stress a parser implementation at points traditionally used for such attacks. However, this document does not attempt to be comprehensive. It should be considered a seed to stimulate thinking and planning, not simply a set of tests to be passed. 
5. Acknowledgements

The final detailed review of this document was performed by Diego Besprosvan, Vijay Gurbani, Shashi Kumar, Derek MacDonald, Gautham Narasimhan, Nils Ohlmeier, Bob Penfield, Reinaldo Penno, Marc Petit-Huguenin, Richard Sugarman, and Venkatesh Venkataramanan.

Earlier versions of this document were reviewed by Aseem Agarwal, Rafi Assadi, Gonzalo Camarillo, Ben Campbell, Cullen Jennings, Vijay Gurbani, Sunitha Kumar, Rohan Mahy, Jon Peterson, Marc Petit-Huguenin, Vidhi Rastogi, Adam Roach, Bodgey Yin Shaohua, and Tom Taylor.

Thanks to Cullen Jennings and Eric Rescorla for their contribution to the multipart/mime sections of this document and their work constructing S/MIME examples in [SIP-SEC]. Thanks to Neil Deason for contributing several messages and to Kundan Singh for performing parser validation of messages in earlier versions.

The following individuals provided significant comments during the early phases of the development of this document: Jean-Francois Mule, Hemant Agrawal, Henry Sinnreich, David Devanatham, Joe Pizzimenti, Matt Cannon, John Hearty, the whole MCI IPOP Design team, Scott Orton, Greg Osterhout, Pat Sollee, Doug Weisenberg, Danny Mistry, Steve McKinnon, and Denise Ingram, Denise Caballero, Tom Redman, Ilya Slain, Pat Sollee, John Truetken, and others from MCI, 3Com, Cisco, Lucent, and Nortel.

6. Informative References

[RFC2822] Resnick, P., "Internet Message Format", RFC 2822, April 2001 .

[RFC3261] Rosenberg, J., Schulzrinne, H., Camarillo, G., Johnston, A., Peterson, J., Sparks, R., Handley, M., and E. Schooler, "SIP: Session Initiation Protocol", RFC 3261, June 2002 .

[RFC3264] Rosenberg, J. and H. Schulzrinne, "An Offer/Answer Model with Session Description Protocol (SDP)", RFC 3264, June 2002 .

[RFC3986] Berners-Lee, T., Fielding, R., and L. Masinter, "Uniform Resource Identifier (URI): Generic Syntax", STD 66, RFC 3986, January 2005.

[SIP-SEC] Jennings, C. and K. Ono, "Example call flows using SIP security mechanisms", Work in Progress, July 2005. 
Appendix A. Bit-Exact Archive of Each Test Message

The following text block is an encoded, gzip-compressed TAR archive of files that represent each of the example messages discussed in Section 3 .

To recover the compressed archive file intact, the text of this document may be passed as input to the following Perl script (the output should be redirected to a file or piped to "tar -xzvf -").

\#! /usr/bin/perl

use strict;

my \$bdata $="$ ";

use MIME: :Base64;

while $(<>) \quad\{$

if (/-- BEGIN MESSAGE ARCHIVE --/ . / / - END MESSAGE ARCHIVE --/) \{ if $\left(\mathrm{m} / \wedge \backslash \mathrm{s}^{*}[\wedge \backslash \mathrm{s}]+\backslash \mathrm{s} * \$ /\right) \quad\{$ \$bdata $=$ \$bdata. \$_;

\}

print decode_base64 (\$bdata);

Figure 58

Alternatively, the base-64 encoded block can be edited by hand to remove document structure lines and fed as input to any base-64 decoding utility. 


\section{A.1. Encoded Reference Messages}

-- BEGIN MESSAGE ARCHIVE --

H4 s IAEDwCEMCA + xdW2 z C2Hm2 nexNG6UN3LRF 0QfaikJdyxwdnkMOhyOPVrIt 27 It 22 tdvHYTeM8MD zWc 4 ZA jkqORvK2bbIAAedmHtEHRdlvkoUCLFA jS lyLF 9rJPLYOWrTdAg 6 JFHwp 0 i +5D 0 S IoEAQFu j2 HnAuH5GgoW3P xmgcazYU/b 4 f / / / 3Xc0 4Rq9ipk1JGxe6xITVAW1YUvXc5K/W8syYheEygP0l IECWJ0gkSkMAx DhwbQWs 4 LrY57phdcerYrjr96AZtb91L5 / 0paTdvbazevLHOOXo933CIvUT2 cK1ukIxlb3Prq7 fmYQZMT23pON/+Nr958RZXthxXzLRpS1YtL4EsWCja2CyV $\mathrm{Cw}+\mathrm{U} 8 \mathrm{mWxeK2qVhoigkicnlrDe/wly25iW3XynEwPecmmO3GnzxPDOMstG/RQ}$ pkrs 0 9w5 diU4s50p0i I LgTMs Lrh 4 uyAi JEI 0PbVh0 Z3vYNexzLP CRtmqYYfu $692 \mathrm{Gm} 21$ 6v/f cyuL01AVs GP zqxTxXbP0802qAXp4 JTdUBGChKA6IyKptmEwCl pFZNQs+0XCqRupvncL1u 6CXs 6pY576h1erx1spPnkALpLSpcqyOnp4w8R29v eurpeP 60L/yHNkQAGCT / IsiG5R8KMJX/sco/lbiu/DNpi6Noi zHbVqLi1Ysf nsAiBEUYBgAUAymCQj9lYEYIochBEhiQ6BYXO1 i 1 TM2CSBChqOwC7AAKKxqq I LMt sbmnVVaHJP 9U8Mkw 1 f 8g+RcAF I +xf 6 I IBJRFVP 7FbDbV/yNpqze2VjdW jl78TeJ64g+pflWYwo5aAEHp9XiQqlGq22 smlWEqsBAZFRHYvENUzax5VoQv

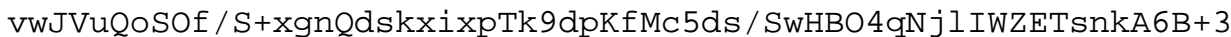
sr5Bz2iZLi5R7DkXuEd2 fCkTuNNFn 5CYLr+xXydxSNXafJ2Y226z3oPk 4 c5u gb5ZhVqZGj8G2eegI INTQOYYvUGF 3 iC 3ekvsAKM0PeUU+OmpUiG6wS0AhmS 1 Am6ousXRLhdk7vbGrfnlrVscvSnItu3qKrE4BGF 3ExKmp3DBdus 1XM8 jgbt+ 68Kz jIbP Jv6bQ0X/BP 6 fgEL $2 \mathrm{n} 4 \mathrm{gkKCX} / \mathrm{Udt} / \mathrm{sY} 5 \mathrm{Trw} / \mathrm{IWhBqS} 018 \mathrm{wGYY} / \mathrm{b} 3 \mathrm{~W}$ dQJpC 7mBg 71AXY 15 rdcL 9HeNu5WQC0 jZnvKbIC313MNAf4+2P i 7f0yr/urkL hHHGf2QEwvafDFAg/xNn/1Uyj2EBCkgUstSiF 6CYjZiBvSLpcy IoY6A 7poqr jlrBDrUFWYwGO13/ra/l1/EhpYWF swtnzwYMuNNXLdKKLlUs0oMLC7 TFmWhw oFl3SAt06GvCCeOy4Wiv7xLbGaf / B0 QxqP $81 \mathrm{~T} / 5 \mathrm{RNpX} / \mathrm{idH} / \mathrm{ckT} / \mathrm{y} 3 \mathrm{H} 6 \mathrm{P} 6 \mathrm{nq}$ 79H8yxlP+Q/S+DtNYuk 7dRLQ+xuZlupP rP I 9 TmdKXW4 r /Y 5uF 5 6x 4FCxhKmz PFb7n4p+JP 6D sqn8j6S1tCcHAeBuXjt IpSq5kHwLCPqhncg+UJIygVd4PwcX ic127Mqmx 4 UA 5 cScCCAQqMKny l /DVVSBxG4 SVXOW11Wtk3OROiZA1/wImya+ 8SFQaUdt dyFCRtRGK0 OF ITgLQEwU2OkGiLyDs / AQzAXxZfHwloKS62sqsE1p vCdtR4P / ZM8drveXIP 4 jS2H7T4RCiv+Tl/+r3H+CFIAI iWuHDCF sEP 59 Juxx /Kanbpodhm5 T0DUtt 6yb2+uNet 2yXWe jrDtn435c0d0yoSe6zVt 7+3xgd/aD TpwWbXt / +6K1b05Ht 8XkCXs 03Mb1dU6 zD JWnQM5T 7vEUySArOKxbJ sWyLOrb JUsda / 4P S I IY8f / S+M9o7T 8RKqKS IREQqD S 6LrGZgHFFm+AqR6WKs 0mJ 6LtM uvpbiCBs 6UGk5Kg 4 WyQo 6y 2Gw 45 qaahRgQDRj 6 aG6BU0 6Keyhh1Eyl 7 gBzuK 3 rX5kKiI IbvvXBxs+Q 4 jUrDpaHrL8 jsXz/r5hAqAFVM1 q6zWJ0zK+xh4 9GYj Ft 7 TqP 9LFLHtMed/5CwM+3/UaE/I f2Liv72aO/ekEWGF 5 fnpPwv2S7A3zG1 7 P 5xhbyOI z 7 I 9xkKT6 Ji i hVpFCYLEven 7qMbmWX5H5CGS IDp0Yl+h7 THiwgcE hocbGS5Rpsa18ez / JCBE 9b+cyv8o2u2Vy 6vrGyu3PXmNF f 6 I / D jYbdjmYyV+ u 5F fdrpQvLYds 71 Y 1ba2K1XbXWtiYl+71g76xu 8SBIY2L1PuEcBS 9Drb4AC5 9m0HAIgdfk5QZEhZENK2tN0UghC0 ODCrptU0vZN8YqLDrT 6D 4 5R/MSt H5B +m +v9Zlf8cylEleTiZhwNlHsXJ/LlDCf3iJzAnpBYNm+yMNf4nICkbtv+ltP 5 r $\mathrm{UuQ} / \mathrm{makf} 3$ doq1 IKSUEEVBCODgHLTU6t $5 \mathrm{rsV} / \mathrm{Pda} 80$ jD fXzMNZFQhQqIAQ2q 6 $\mathrm{dbJwnW} / \mathrm{X} 9 \mathrm{u}+$ Kev9oBZTiEMsrV+4 TrqMX3PWWgkUkPf3Vvsbe7UkKZajTi+K6 qQN2 4 c5SZJnKleJJ01KwlOQwhT IxnYBywK+3Hn5R850XxHCJP Z $800 x V t \times C k N$ $\mathrm{O} / 0 \mathrm{zOP}+\mathrm{P}$ 5DD+wz T+M/L4D 30 5M2 i ZQeuMCALYFUSqqF 6YkSWHzMgwDu 0 8+2p1 BlLE2iX0 jXZhiTjB47VisCgXwT15ekrPcz5/spEhQPFCwtVK1YTIMukXwKPA sBDIBoaKScM+DHT jEzUHJPmnp 7hOnGomeyFuKMgC / Z12xoI 5kxVqpLBL34 wG 
vXVpBokzSFg8ItTsC5ppaUDaDov/cMzx/2zU/6eSnOL/aOz/GVGmtvKMKPk+ gE22dce1sz3p 6w2 cnrlHyVvF1DZxrIZd6 jF 2F zvD+wdSevCvQQQrWNUqPUVh Pmsy0Dd3mhYS 7R2 IdCRWbJYbLJkGRKZtVE2H1YX1 JugvDBwDCIEyoz1wTGIE NYiCRJEL $9 k$ js sMWe 4AE2 dOwI fkowlBC8MJO 9FCGF fnlYmDR6TOQRohDhkccf aCebDcMYb / 5 f jMb / sun 4 / wn z / xmb 8DMA 80xDP 9e2L1Ersqco 0 J+ / 4 D hwG2W RAOEYFS13WpFxY5W3UFN0XLtHYBVR6OggHfVzpBgESk 5 Zv0d4PgP SvsFCnW6 okhvZSmy 42 IMVT / C6/nJjhfSzrYbt j7e8f 9 iJP 4nS6n+H7X/Fw7gvXbbarik MIMuhLBhBq0 cydwASBQk IRc 3 Jqz fqHsPP / rVBbRZ2XMWeWY3l Cs 8 rhBUt HmK DTtAyTV5DTeJXYY7fFk0pS58UKihyh8e7D3ylsa 7ZcKXqRmTVOJvmMGz1A1M 25M13XQa2pj9P zEbGf 8 rpvnf seN/F+NbKOnVbQ3OKSgxOYWMx2 cDQdFoDbs 9 JA4qfZMIS jo3yiD5d2vELY/V/ OM99V9z3/5L/b+RtFOUAYhNHFc3t/k1ygmW 6g2/k7JyTrl/Zu7Nz IxuqoSosw89kBDuN8yG0 8BGZvP26sNXXIsvklNOwyav flF3zFl3Tne/MF+y8YP $91870 L y y c y X 9 R d+f K 2$ CkIZ5tEt 9VTZ+rY+ULTeqje dy0r84pqEbYbr 7uvLg0uP2 l HdoSDy jczp2ay2TP3596 ffikV51 fuz 7 / 0 gvc jU36doy 7yL79ai soddj7iQ1 zuVaVmMLDN / 0P cHbuvv8 JnZk5leH9E9UaporN UPBLo 7vfYqUls 7MP 587 cp8QzhdMffOXR9x790aM/ / +DtR9/ 74 J0PvvHo+4 / + 5LRnMN / 3 jvpQmJ17kx6z zwSM3 7YCNy1bnb13jT+VoT0wu8S+vvnw1VcWz+W/ $\mathrm{NH} / 6 \mathrm{y} 7 / 02 \mathrm{q}+8 \mathrm{FZhGS} 4 \mathrm{AQ} 5 \mathrm{STuEDwQNt} Y \mathrm{hK} 08$ lexTTHQriVwhWiU3PPXNm7 j7t /vx/vfcXH/7e73/41Xc/+u33JncMzLNt/+1CSURjjf9lvfF/IfsvHf83avtv $\mathrm{k} 9 \mathrm{p} / 55 \mathrm{eS} 1 \mathrm{QDqmrvdzPTL}+\mathrm{M} 4 \mathrm{JCCBJkgTal}$ i hppmToVPB 7C+vo2QrismWSRTbU rOSBivCCDq1 jRK5moYZV1S $44 \mathrm{~T} j$ jO305AzAlZCbTr+IJkvafrAU2f+QVZkOOu M1BT JGU7hu/Rzgnz+LP 6HQ120 i 5ouOPO/wE5bP+J6fyfk+T/JZ0F 8 4mGBew8 gL94 YG7AZ 9 feGaJUR 9MrbBZvpHZrQSRRPKD 8 zbF qJNk s of 2 FvVUZrF 12DbtR 40 b0rJtznuTSnuHO1Uu1Bs fGGBdOiy I 6PU9/PDff 3 jy 2529 Y 5 vawC 4 AHyH 82 K6NI/D/N/02Q/HtO1CrHirg4zDE6zsQ1wlkaR21/m9TIE75xddtiokHl6nTs mN58lvZpTzG+UNgl9j5j36N87WKjQRbYJ+8k 7C6H/So4ZXrn/omHcUtxL8/n JNTpu OHf7uhu+Ya1xS6AebQ+RuMafkDdQc07HB+w2 cUO8+doQTRUcpP 4 J0 Kx zYplz+MdCq8U/FMEzuDxyNH8a1+/99QN4 jidWz jyDwHt3VY21Aq3Cf1xf/T / 2yynd2wFlGOVA6BjLGz 6P cNfp5RH+eKZrOW5VsfzRy3SvP 9 ch 3 f 8 ehs zeA/ 7 C6M4k3dPMTFAilAI 9bppu1EK2EuxFaUQQhRx5wFhmuUIDVRCNKvR4 / TOCMZO Yo4 jh+4p5npgNkwT cxwpRBN3PWKY JuY 4 oT 7e 4vJchCbUy 7 txNOF+5r jouUD 4 OFEaQYk 8 rxi iXJQohkoe/OiFbAIaKQGNmIAGJaCBCWgS sLQABtMog0lygoks dHKCP k 7QxQ160EEHJ+nfASQRr7I1CY5a 6MR1207mqIy9Ubz 8W2 rB9cCa2 THY lo+xZofxLBT 1GO 620+wCwIHz / 0 Tmf 5WEtP 5vpP 5 / ERaR1P lp0BFiOQNg 4X+ HR4UlgLB71aWenC9i TTMZXqUIw70I OFUEMz JYEKAwGg 6qu 7Ux $5 \mathrm{r} / \mathrm{QzKL} / 7 \mathrm{OB}$ 3 jKbDNqP / 6X1XyNpR7P + ny 9x52 JQoDsf34 Cq/zYjss IDXCypSxr1L/f jUnFz 0GdiTgYKkb3iw/rpwn9d+R9//T8K5/81ANL5P 8Yd/1/gDHswBP jCvacRXqFK LJdD / ANF S z I rMP JnZo/k+6ReUPC 4058 MVLWIpbol $17 \mathrm{zi} / \mathrm{qZt}+\mathrm{p} 9 \mathrm{ySLSr} 3 q \mathrm{q} i$ VvJPQNSk z IooQa2q0HfqIoiUgwwLYfSGxCGOxaQZFml 7WvCfAaA7Vv9PhhH / T07rP0aJ / / H2X98ZYJ/I I cz IBLEX41s cqFXNHWr9UYwXEVAUrLKh37hJ0FKM FS jkFJTLDZjvQxhkCSKlPCfrCFB+4sNHtZIx7vl/gCRnw/Vf 6 fwPE+X/HXoy HTaVAkT JYMJiqzbEhY3YCKMAUP i sqpVNZg Jat 1 7GTU21MLJEW4 IW0m2 nu 0 IQ ta+o+ddxEyOCGfFF syBKJYUF 3iV7 7nzdwLrJxHqDXjaZdT jT 4pp 4n 3Mt juVD adc0uRo2 9 zmr5BLX4bBNOI et LuEQlREVCcd2 zEyG+1nTnRp2S+VhgsDA+I 8k huRfgmn 95yTGfx6rrhOJEpQOWv 4 ly IMVNvOgs 6 dqRtKp3NNgz $5 \mathrm{HIPxy}$ / Mve +L8e+59+SeV/FO3Gyp211Y0rNy9OBgLAAQjQ11 IPGeoHI0X/y f8GZ4TZTIWH $\mathrm{N}+\mathrm{nj}$ jPmJiNHUKFMP ewG 4 Af IPRYH $5 / w j K 9 M k j$ MM3 / JKX 6 f zRtbWV9f fmyHwCu Nmp 61Yqs/hvvAQhQpowMvAiqHI 6g 8mpOlkTEiypWCCiqoKgWBYEQXuDbmxZs 
VvAdlxIKjgILniUHqGCvqlQ+dXc/z 91SrWmT6439i 7 fvnt fnwYZ+q7ni 3EX2 ypzTnb 8M7+yVt ze2hFuX5P KVxvXVa+Duna1r98rXdpW1Sm6TvLFTXds qmls 7 SL5wCS 4 noo3du 5XS+U18 z 9x+vfZGSbl z $4976 x$ RuXLzxYntPuGMrayuaVo6v 3 LOECuUqK6t 35N4 zta2QDg5vG7g6wXRm8LlX0da24s3a19vollxB9d7NcXbtz d305EAYuzWho2v5Sbx8uBpaqi j6KxZ4Rdq0DRLJsUCsCtVRSAtiKVEXMSVgF JVHJSUpx 6V5tT7hXuyddvyOU 7+6DvRu 65BTh jXJxOZPppt 1bjBHARJst qEVs fsUsWapubrO1vExs 74 dhs 9YwXJ3BynxN3yPqQtFiQ5/t/YKMIVKRIAAsEaJl 2cjhi5g96XXsnqUeG3ez 5LJ1aCUOiHlRzEtZ7vLaxvQUGwnIL2/TE3gcqNfq rD/mvZQ690qpYTVEeTXGe5MQQ2qe j562v1eX4Ganp64Qw7CSHDmoQDxvkHdc m7DVQxKdB7x9/PbzL535+pWv/3j6+Asnvv328Qv0p9dOHD8uvAReeP65uc9+ 6sQvgJe 7 JMeFt 49 z lOQXKQn 4 ZVAXXgYvPf+pzU8 / f / LE5 rpwEnyOfXnxJOMN XbNsU8fC58BPsR9fOPniOja5q9RJFT 4 LPsN+mjr5HOU0 3RVeBbPs+8snuXX2 ndsgjstdILara+zmCLfCcMvUH 3 f3T $7 \times 4 / F v H d k 6 g 4$ ye 7 l z fdubz jx 5879 umv $\mathrm{fuWd} / 9 \mathrm{~F}+\mathrm{cuW} / \mathrm{b} / \mathrm{M} /+\mathrm{PA} 33 \mathrm{vmdb} 33 \mathrm{nox} / 93+\mathrm{e} / \mathrm{rd} 3 \mathrm{dKX} 7 \mathrm{zP} / 7 \mathrm{pzB} / 80 \mathrm{zRV} / \mathrm{ruP}$

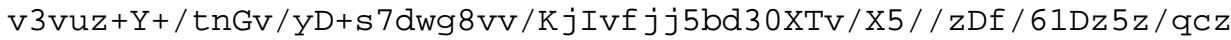
b $9 \mathrm{nf}+\mathrm{MG} 77 / / 1 \mathrm{dzP} /+1 \mathrm{fv} / / 1 \mathrm{Pfm} 5+4 \mathrm{cTDm}+/ \mathrm{P} / \mathrm{utG} 7 \mathrm{~d} /++\mathrm{Dv} 59 \mathrm{xa} 1 \mathrm{v} / 2 \mathrm{bL} / / \mathrm{w}$ L6997Ws/8+73//3jn+d//eaHb5E//c1Hz8U90J5/Vr0DT1jHq/8FCILjv0Rf / 6 frP $401 / 0 s 1 S R 2 b+w n H f 0 J p k N 3 d t n f j V 1 u I r Q j x x 1 t 3 T f 60 k 9 B i V S x H l o l c$ Cl jSrV2UxPvAA5yWTk6bpafZIgT9Y5 jtLZEIZpL8t zcT JptnI1khqn jet H Z OGOIZNQP 6bHAvkVs 8KgnVErDMmNtZskYCVYnQfynZ / 5PD / 9BWv83HvxPsP 4n ZFj0 ZPEgL 7WDgl lgxoc 9w/ gh 4 KWQbEmETdQpBWY JyoFRkol 9e 69MQk +W6OUf Z9VP 5p/0qRRGIXsqYFrUIXEsc6zr/0EYYP/IXv0HSuV/JK2 9/jftf+6J1/bu MBOmUsxW9tbz7PyKAiGbx7u7kne6ivfk6H9rlxhOaaz1/yKUQvKf1v+PPP9r 4XqmSEg 9P z / fFXqURwDAoxkGl ItU+qIe 6PD50K/0pYcHOxRhzKZf+Fs luqt 0 IH8JgT5 jANpjMJUklV9iOifhTsNyi3i 863 /Iohs2/+V0/c8Jsv+9+X/W7Ax3 NCOxaLyvRaOm/2 ICHFBQzCKXQnAkUJs lPT f goLF 9SthoT 7 rqpaTkJSCBBLCQ uNAT jmHly6P IEdtkGzeGnABONv97r/4X0/k/R9KGvP 5vTkZQKF cgEmDuoJVt O2zYEWNoizTF jL5r+jKF3w109vH9WxmqB54 I57kb1k2TXDOtprNR1p31UplN QWbV8U6D 8FQOCIEVaEak/BNpGtCOL2K15I5X/l HY/wdp/mfS5P 8g6Y+VfUXJ IQQP HP Tb 4b 4 qE iXs 2AbS 1 OLukgD 8 sohuNcJBC30 jKSP kKDViL 9R3QF 9oD fGD Vzuir0zvikef0DJS+gTYuknjlX8pG43/pfWfT7v85waJvs 94 IdEPZ3X/v72j 2W3bBt 8L9B2 IALsEcUqRki 05c9F i2LB2a 7 I 169 JDgUKxKFv+oRxSspqctp $4 \mathrm{H}$ 7F2G9bjtFZI3Gj9RdiXZlpN2tZNVbFLHICjJ9Pf/+x+29nu2wt S4DvXvQBu / Dx5SKVSu+LQtgNfq/9RckP 9Jjf+3BP+XN2AhVQY2ahLp+eFqs 33eVlBh/Seg jZNBf $x 4$ I Tpu2Q01 I IFUKogMxw5 TkCMO cuMyhmtAQT zxn 6vLRGU2 4 GHGWhP 7 A IU 4 cLMomFKvP + /WbSSBYapTYwF jtmi juMJQQRwdMP 1 uH 2 Jg 4 LctpNimxDes j z Jh6p0a 9beJ/U+d/ENLEoCim8n9t/9us/8/CFB0 zMQ26DL3g7tQNRu7piH0Q +19cvPEC 6Ngtk8Xyh2 rptanCQlxVRhaYK72BzwbJ3EBA 0mxRm5qt FqHYhEQC I 5 / / WSQMCt X 5 6EqGQx+ST fqcn IV+6MIb 4 rLEh2 s J6EoSifPGY z 9iQt 3CdCgm NrXVTsErvIX7OLaSc 0 5cwdP gXcNWtw2nTESXYG jnCUc/i LCnUFbu 3E $5 \mathrm{fg} / \mathrm{Qm}$ $\mathrm{W} 8 / / \mathrm{blkL} 8 \mathrm{~T}+\mathrm{E} 1 \mathrm{vr} / 7 \mathrm{bL} / \mathrm{p} 0 \mathrm{a}+14 \mathrm{tKwOraCeXY0GuUBKosBqRBNTMNQnCQp} 8 \mathrm{iA}$ d/ /e426XTaIs jp+Hp6F3Prft3cApYF jVOWGFog 63pQfQRz sCJBsHW67/ha1W Tv5vZvX/mjX+b2KU878P I IfsXF+YOIecNycKK1E9FD0dDUCJSariAWZwudQf YL/n7DPkL6HgXmF iMuzKVmMc jNkeiNF 6 lNKU9nITMuhxNZM7VzFwt 8 fUAXXl 3BtfuL0qknL789EjJj91+6/19r98/ReN/6RV+/82 zf+VZMwfKdU4HAdZesMR UohVntW4 LdzRpF 9E+mfuy 4 / JE7c596xgnDczDv4UjgrIHPe1P fy/OLivEHU aYpnI/WjBH/E4 /EpE2OlRzpCQJ9F23Nenk4UFHX1FMzVorgfhuhUfbXqa 40n DkN4 I 9KXJUzdvi5P j3Sf1E6auHH37X8RJCBCJq7cYv2HZquE/5ZV2/+3w/8/ 
rP7vR1X+RTkYHAaDvukOPe6eDYYJ4550pQ+MfwWfv1ZN4PVl4EqLjr/6Sa0i VatGrhx $4 \mathrm{fb} 9 \mathrm{sH} / \mathrm{n}+$ GEW0Yh1 xbBxzDz5R8TEPvzs $80 j l \mathrm{EkVmxWKk} 72 \mathrm{KeMLzHK}$ VLo/sOsEns 8ZWyJ6RCKR2+b/Fi7jv0lxjf8bx3/BxmHEGmo/esuFfoQq+7Gv gHhQ1bGT 9wWsvFHR/DCKFUlpCCbDWHTZMs LimMTBdr7aQQrPYdCNqel YNoQQ Mx 8 iiPsCGpqLtYSEUNvJBXCV72Xy/4kYjvlQdseflgKslf 9NWsb/Zo3/m8X/ kt 1Mx8S1ozCCpnk6NK6rAX8T2QAZVPI 6G2AT+D 8mW8Z/Ssr4b5Fa/781/n/A $8 \mathrm{kCe} 8 \mathrm{raSXWnLIXaLzrl0P} 4$ ogYShJkv1lYr9CzDJWp7CWqv / $980 \mathrm{mfCTdiXqNC}$ xVE9MzYdnH fwL3Lj1QUnjOtEAn 4 JzcHaT 8M+f308DqL+w8+tWmTMt 57 / a 2Cj 7P+zDFrXf9vImEn2BGPUQWR3 $16 \mathrm{JdZO} 3 u E n T 51+X f 60 r X q 7$ fq5 f LPy 3 eXf 1 z 9 DnOlqau3V7+hBrr85+qX2bHLd1U2 cccu 4 aRBiemQI qHI gBKaAQ2B 6Q/pgDCR $\mathrm{MM}+\mathrm{Xj} 48 \mathrm{rU}$ olXBiMahm43skJqYV6sXZAEwhMWXX7XSwlaFsbj2AsZxjdLE3Ls IXICCOj2tRSsxx0aigz 4dLvxH0bLMsrxH0oArOn/hv0/02DKhr1H3b 4 bi IbH RbegNGXdvtPe3Dnh8Ai 179 9bvxwdI IQUgQVnakcRMMOGf5Ty+ / d8AZ1GUBvt PEXPQ8n 4KRM990rVq52dzPuqs0 0 9AQCfLCREwn9w5Q6CII 8Dz17jidIQv8QJ VaT66SOlsR5dmVaewdiBW+XiSLK20gg 9UCen24GA1Wm38VwOnXE76mCZiZ8S $\mathrm{Nu} 2 \mathrm{QJd}+4$ vDdi3rfM9SDEDCHOEl / PoayXLgKtO+Cxmlk8mLWq1+tPlP j6gscy $\mathrm{dkc} / \mathrm{w}+\mathrm{E} 20 \mathrm{j} j \mathrm{Y} 210 / \mathrm{B} 2 \mathrm{t} 51664$ qm6W7rTUYYCy $8 \mathrm{PWP} \times \mathrm{AwCEnWf} j \mathrm{~m} 42 \mathrm{P}+\mathrm{sAz}$ 0Pd qf 1 h 20 Z 9 ti epw fABAh 0 chpwEwO 4 KUJF 9 fXqHAD IQyoQCJxgObITABz / fYn $1 \mathrm{~F}$ 9SfIE+kGG017n1 jWvuLucK3sQh21 aBaJDBF 6igO2d36MQ6VqIBmJgCvw2gHw WglY6lJqtzWsd2 ZhAGom/ZT6mSXYQzx9ygE6U8+09ym9MXt fWQP I 3Axrv2AK /fwt 6/8EL9r/av3/Dvn/qix9vb70TCNHgDOQG4Apb+TzMQnJyKTCirE2 9xVM e5QYFZ69a/V4AitCULYd4Lr0Rz3qUY/P fPwLcaRGXQDwAAA=

-- END MESSAGE ARCHIVE -- 
Authors' Addresses

Robert J. Sparks (editor)

Estacado Systems

EMail: Rjs@estacado.net

Alan Hawrylyshen

Ditech Networks

200 - 1167 Kensington Cr. NW

Calgary, Alberta T2N 1 X7

Canada

Phone : +1.403 .806 .3366$

EMail : ahawrylyshen@ditechcom.com

Alan Johnston

Avaya

St. Louis, MO 63124

EMail: alan@sipstation.com

Jonathan Rosenberg

Cisco Systems

600 Lanidex Plaza

Parsippany, NJ 07052

Phone: +1973952 5000

EMail: jdrosen@cisco.com

URI: http://www.jdrosen. net

Henning Schulzrinne

Columbia University

Department of Computer Science

450 Computer Science Building

New York, NY 10027

US

Phone: +1 2129397042

EMail: hgsecs.columbia.edu

URI: http://www.cs.columbia.edu 
Full Copyright statement

Copyright (C) The Internet Society (2006).

This document is subject to the rights, licenses and restrictions contained in BCP 78, and except as set forth therein, the authors retain all their rights.

This document and the information contained herein are provided on an "AS IS" basis and THE CONTRIBUTOR, THE ORGANIZATION HE/SHE REPRESENTS OR IS SPONSORED BY (IF ANY), THE INTERNET SOCIETY AND THE INTERNET ENGINEERING TASK FORCE DISCLAIM ALL WARRANTIES, EXPRESS OR IMPLIED, INCLUDING BUT NOT LIMITED TO ANY WARRANTY THAT THE USE OF THE INFORMATION HEREIN WILL NOT INFRINGE ANY RIGHTS OR ANY IMPLIED WARRANTIES OF MERCHANTABILITY OR FITNESS FOR A PARTICULAR PURPOSE.

Intellectual Property

The IETF takes no position regarding the validity or scope of any Intellectual Property Rights or other rights that might be claimed to pertain to the implementation or use of the technology described in this document or the extent to which any license under such rights might or might not be available; nor does it represent that it has made any independent effort to identify any such rights. Information on the procedures with respect to rights in RFC documents can be found in $\mathrm{BCP} 78$ and $\mathrm{BCP} 79$.

Copies of IPR disclosures made to the IETF Secretariat and any assurances of licenses to be made available, or the result of an attempt made to obtain a general license or permission for the use of such proprietary rights by implementers or users of this specification can be obtained from the IETF on-line IPR repository at http://www.ietf.org/ipr.

The IETF invites any interested party to bring to its attention any copyrights, patents or patent applications, or other proprietary rights that may cover technology that may be required to implement this standard. Please address the information to the IETF at ietf-ipreietf.org.

Acknowledgement

Funding for the RFC Editor function is provided by the IETF Administrative Support Activity (IASA) . 
